\title{
๖Relative Dispersion on the Inner Shelf: Evidence of a Batchelor Regime
}

\author{
Matthew S. Spydell, ${ }^{a}$ FAlk Feddersen, ${ }^{a}$ AND JAMIE MACMAHAN ${ }^{\text {b }}$ \\ ${ }^{\text {a }}$ Scripps Institution of Oceanography, La Jolla, California \\ b Naval Post Graduate School, Monterey, California
}

(Manuscript received 28 July 2020, in final form 17 November 2020)

\begin{abstract}
Oceanographic relative dispersion $D_{r}^{2}$ (based on drifter separations $r$ ) has been extensively studied, mostly finding either Richardson-Obukhov $\left(D_{r}^{2} \sim t^{3}\right)$ or enstrophy cascade $\left[D_{r}^{2} \sim \exp (t)\right]$ scaling. Relative perturbation dispersion $D_{r^{\prime}}^{2}$ (based on perturbation separation $r-r_{0}$, where $r_{0}$ is the initial separation) has a Batchelor scaling $\left(D_{r^{\prime}}^{2} \sim t^{2}\right)$ for times less than the $r_{0}$-dependent Batchelor time. Batchelor scaling has received little oceanographic attention. GPS-equipped surface drifters were repeatedly deployed on the Inner Shelf off of Pt. Sal, California, in water depths $\leq 40 \mathrm{~m}$. From 12 releases of $\approx 18$ drifters per release, perturbation and regular relative dispersion over $\approx 4 \mathrm{~h}$ are calculated for $250 \leq r_{0} \leq 1500 \mathrm{~m}$ for each release and the entire experiment. The perturbation dispersion $D_{r^{\prime}}^{2}$ is consistent with Batchelor scaling for the first 1000-3000 s with larger $r_{0}$ yielding stronger dispersion and larger Batchelor times. At longer times, $D_{r^{\prime}}^{2}$ and scale-dependent diffusivities begin to suggest Richardson-Obukhov scaling. This applies to both experiment averaged and individual releases. For individual releases, nonlinear internal waves can modulate dispersion. Batchelor scaling is not evident in $D_{r}^{2}$ as the correlations between initial and later separations are significant at short time scaling as $\sim t$. Thus, previous studies investigating $D_{r}^{2}(t)$ are potentially aliased by initial separation effects not present in the perturbation dispersion $D_{r^{\prime}}^{2}(t)$. As the underlying turbulent velocity wavenumber spectra is inferred from the dispersion power law time dependence, analysis of both $D_{r}^{2}$ and $D_{r}^{\prime 2}$ is critical.
\end{abstract}

KEYWORDS: Ocean; Continental shelf/slope; Sea/ocean surface; Dispersion

\section{Introduction}

The turbulent dispersion of tracers is one of the fundamental problems of physical oceanography and is relevant at a wide range of scales. Tracer dispersion can be quantified from the motion of particles advected by the flow. From observations, estimates of oceanic dispersion have been calculated on the mesoscale ( $\approx 10 \mathrm{~km}$; e.g., Zhurbas and Oh 2004; Rypina et al. 2012), on the submesoscale (0.1-10 km; Poje et al. 2014), and within the surfzone (10-100 m; Spydell et al. 2007; Brown et al. 2009) using surface drifters and subsurface floats (e.g., Rupolo 2007). LaCasce (2008) offers a thorough review.

Particle dispersion can be studied in the framework of absolute (single-particle; Taylor 1922) or relative (two-particle) dispersion. The pioneering work of Richardson (1926) investigating the relative motion of particles within isotropic turbulence established the foundations of relative dispersion. These results were later theoretically justified by Obukhov (1941) using inertial subrange theory, i.e., a $k^{-5 / 3}$ turbulent wavenumber spectra (Kolmogorov 1941). The essence of turbulent relative dispersion is that as particles separate to larger length scales, larger more energetic eddies are more effective at dispersing the particles. For two particles separated by the distance $r$ in an inertial subrange, this results in a mean squared two-particle separation, or dispersion $D_{r}^{2}(t)=\left\langle r^{2}(t)\right\rangle$, that increases in time $t$ since release as

Denotes content that is immediately available upon publication as open access.

Corresponding author: Matthew S Spydell, mspydell@ucsd.edu

$$
D_{r}^{2}(t) \sim t^{3}
$$

Because the relative diffusivity is proportional to the time derivative of dispersion $\left(K_{r} \propto d / d t D_{r}^{2}\right)$, (1) is equivalent to Richardson's 4/3 law for the scale-dependent diffusivity $K_{r}$ :

$$
K_{r} \sim D_{r}^{4 / 3}
$$

The classic Richardson-Obukhov (RO) scalings, (1) and (2), do not depend on the initial separation $r_{0}$ (e.g., Salazar and Collins 2009), hence the coefficients of proportionality in (1) and (2) will not depend on $r_{0}$ but rather depend only on the energy dissipation rate $\bar{\epsilon}$. Thus, RO scalings are only valid for times $t$ after release large enough so that the two-particle separation vector $\mathbf{r}(t)$ no longer depends on the initial separation vector $\mathbf{r}_{0}$ (e.g., Salazar and Collins 2009).

Batchelor (1950) considered two-particle dispersion but for times $t$ just after release such that the separation $\mathbf{r}(t)$ depends on the initial separation $\mathbf{r}_{0}$. From dimensional considerations and a Taylor expansion of the velocity field about one of the particles (e.g., Ouellette et al. 2006), the perturbation dispersion $D_{r^{\prime}}^{2}=\left\langle r^{\prime 2}\right\rangle$, where the perturbation separation $r^{\prime}=\| \mathbf{r}(t)-$ $\mathbf{r}_{0} \|$, grows ballistically in time

$$
D_{r^{\prime}}^{2}\left(t, r_{0}\right)=\mathscr{Q b}\left(r_{0}\right) t^{2}
$$

The Batchelor regime (3) is only valid for times less than the Batchelor time

$$
t_{B} \propto r_{0} / \mathscr{C b}\left(r_{0}\right)
$$

(e.g., Salazar and Collins 2009), the time it takes the perturbation dispersion $D_{r^{\prime}}^{2}$ to grow to the squared initial separation $r_{0}^{2}$. 
For $t \gg t_{B}$, memory of $r_{0}$ is lost. A different Batchelor time scale $\sim \mathscr{C}^{2} /(2 \bar{\epsilon})$ has been investigated (Bitane et al. 2012). However, in an inertial subrange where $\mathscr{C}^{2} \sim\left(\bar{\epsilon} r_{0}\right)^{2 / 3}$, this time scale and $t_{B}(4)$ are equivalent $\left(\sim \bar{\epsilon}^{-1 / 3} r_{0}^{2 / 3}\right)$ within a proportionality constant. The Batchelor scaling (3) is well established for laboratory and numerical experiments (e.g., Ouellette et al. 2006; Salazar and Collins 2009). Ballistic dispersion $D_{r^{\prime}}^{2} \sim t^{2}$ can also be found for particles released in pure uniform shear flow, however, the addition of small-scale turbulent diffusion causes $t^{3}$ growth (e.g., LaCasce 2008).

Squared Batchelor velocities $\mathscr{\mathscr { b }}^{2}\left(r_{0}\right)$ are equivalent to the Eulerian second-order velocity structure function $\mathscr{C b}^{2}\left(r_{0}\right)=S\left(r_{0}\right)$ (e.g., Salazar and Collins 2009) where the structure function is the mean squared velocity difference

$$
S(r)=\left\langle\|\delta \mathbf{u}\|^{2}\right\rangle
$$

and $\delta \mathbf{u}=\mathbf{u}\left(\mathbf{r}_{2}\right)-\mathbf{u}\left(\mathbf{r}_{1}\right)$ is the velocity difference, or increment, between two locations $\left(\mathbf{r}_{1}\right.$ and $\mathbf{r}_{2}$ ) separated by $r=\left\|\mathbf{r}_{2}-\mathbf{r}_{1}\right\|$. For isotropic, homogenous, and stationary flow, Eulerian statistics of (random) initial separations $r_{0}$ are equivalent to Eulerian statistics of later (random) separations $r$ such that $S\left(r_{0}\right)=S(r)$. The structure function characterizes the spatial structure of the velocity field and is an alternative equivalent to the velocity wavenumber spectra $E(k)$. In a $k^{-5 / 3}$ inertial subrange, $\mathscr{C}^{2}\left(r_{0}\right) \sim r_{0}^{2 / 3}$ (Kolmogorov 1941). Batchelor scaling $D_{r^{\prime}}^{2} \sim t^{2}$ does not by itself correspond to the background wavenumber spectra, rather that must be inferred from the structure function $\mathscr{b}^{2}\left(r_{0}\right)$. Although structure functions, like wavenumber spectra, are fundamentally Eulerian statistics, they can be estimated from Lagrangian drifters (Poje et al. 2017), but doing so leads to significant biases (Pearson et al. 2019). Thus, unlike Eulerian derived structure functions where $S\left(r_{0}\right)=S(r)$ due to flow stationarity, $S\left(r_{0}\right) \neq S(r)$ from Lagrangian observations as drifters tend to preferentially sample regions of convergence (Pearson et al. 2019). Furthermore, although it is possible to infer Eulerian wavenumber spectra from structure functions based on Lagrangian data for nondivergent flows, the spectra can be inaccurate due to the filtering inherent in the transform (LaCasce 2016).

Some previous research has suggested ballistic $\left(\sim t^{2}\right)$ growth for the full dispersion $D_{r}^{2}(t)$ (LaCasce and Bower 2000; Haza et al. 2008; Ohlmann et al. 2012; Dauhajre et al. 2019; Romero et al. 2013). However, a thorough examination of perturbation dispersion $D_{r^{\prime}}^{2}(t)$ and the Batchelor regime for oceanographic flows has not been performed. In contrast, Batchelor scaling has been thoroughly examined for other flows. For example, various laboratory (Ouellette et al. 2006) and numerical (Sawford et al. 2008; Bitane et al. 2012) investigations of turbulence show clear evidence of $D_{r^{\prime}}^{2}=\mathscr{U}^{2}\left(r_{0}\right) t^{2}$. Additionally, dispersion for an atmospheric simulation suggesting Batchelor scaling (Haszpra et al. 2012).

Early oceanographic observations of scale-dependent diffusivity were suggestive of RO scaling over a very wide range of length scales $10^{2}<r<10^{5} \mathrm{~m}$ (Okubo 1971) implying a $k^{-5 / 3}$ wavenumber spectra. In the Gulf of Mexico, two-particle diffusivities $K_{r}$, derived from many drifters show clear evidence of $K_{r} \sim D_{r}^{4 / 3}$ from sub- to mesoscales (200-10 - -m length scales;
Poje et al. 2014). Scale-dependent diffusivities were also found for 10-100-m surf zone observations (Spydell et al. 2007). The dispersion of drifter separations is also consistent with $D_{r} \sim t^{3}$ in the Gulf of Mexico for $t \geq 10$ days (Ollitrault et al. 2005), in the North Atlantic for $t>1$ day (Lumpkin and Elipot 2010), and in the Nordic Sea for $2<t<10$ days (Koszalka et al. 2009).

At the same space ( $\leqslant 10 \mathrm{~km})$ and time ( $\$ 10$ days) scales for which some observations have found RO scaling, other observations suggest $D_{r} \sim \exp (t)$ and $K_{r} \sim D^{2}$. As dispersive scalings are linked to the underlying wavenumber spectra (e.g., Foussard et al. 2017), these observations therefore suggest a steeper $k^{-3}$ wavenumber spectra at these scales implying a 2D turbulence enstrophy cascade (Lin 1972). For example, LaCasce and Ohlmann (2003) reported $D_{r}^{2} \sim \exp (t)$ for $2 \leq t \leq$ 10 days and $r_{0} \leq 10 \mathrm{~km}$ using drifters deployed in the Gulf of Mexico. Drifters deployed in the Nordic Sea also suggest $D^{2} \sim \exp (t)$ for $0.5 \leq t \leq 2.5$ days and $K_{r} \propto D_{r}^{2}$ for $D<10 \mathrm{~km}$ before dispersion transitioning to RO scaling (Koszalka et al. 2009). Relative dispersion in the Benguela Upwelling Region shows similar enstrophy scaling at smaller space and time scales which then transitions to RO scaling at larger space and time scales (Dräger-Dietel et al. 2018). In these studies, the dispersion $D_{r}^{2}$ rather than the perturbation dispersion $D_{r^{\prime}}^{2}$ was examined, potentially obscuring the proper dispersive scaling (Ouellette et al. 2006). In addition to only examining $D_{r}^{2}(t)$, many studies only considered one $r_{0}$ (e.g., Ohlmann et al. 2012; Romero et al. 2013; Dauhajre et al. 2019), precluding an examination of Batchelor scaling which depends explicitly on $r_{0}$. As the dispersive scaling is directly linked to the turbulence wavenumber spectra (e.g., Foussard et al. 2017), determining the correct dispersive scaling is critical to properly inferring the turbulence responsible for the dispersion.

Although dispersion is generally investigated from the perspective of turbulence, nonturbulent motions can lead to dispersion. For example, the effect of internal wave processes on dispersion has been examined (e.g., Young et al. 1982; Suanda et al. 2018). However, in these studies vertical tracer or particle motion is also required as the enhanced horizontal dispersion results from vertically sheared currents. For surface trapped tracer, convergent motions at the surface lead to spatially localized tracer concentrations (e.g., Okubo 1980; Maximenko et al. 2012; D'Asaro et al. 2018). For various flow situations, various clustering rates due to convergent motions have been estimated using a variety of methods (e.g., Huntley et al. 2015; Gutiérrez and Aumaître 2016; Koshel et al. 2019). How convergence and divergence affects surface drifter dispersion is not well understood, however, the presence of convergence/divergence may affect the dispersion relative to RO scaling (Cressman et al. 2004).

In this paper, surface drifter dispersion is examined for drifters deployed in shallow continental shelf waters $(h<40 \mathrm{~m})$ off of Pt. Sal, CA. The dispersion is examined at short enough time and length scales to resolve Batchelor scaling. This paper is organized as follows. First, two-particle dispersion statistics and structure functions are defined (section 2). For example, proper definitions of the dispersion $D_{r}^{2}$ and perturbation dispersion $D_{r^{\prime}}^{2}$ are provided. The in situ data are described in section 3 , i.e., the multiple drifter release realizations that 
resolve well the short times $(\leq 4 \mathrm{~h})$ after release. Relative dispersion results, both $D_{r}^{2}$ and $D_{r^{\prime}}^{2}$ and their relationships, are presented in section 4. Results are first presented for experiment averaged (or realization averaged) statistics followed by results for each particular release (or realization). The results are then discussed in section 5. First, the role of nonlinear internal waves on the dispersion is investigated (section 5a). Second, how the results presented here fit within the existing literature is discussed (section $5 b$ ). The paper is summarized in section 6 .

\section{Background}

\section{a. Two-particle dispersion statistics}

Here, we describe the two-particle (relative) dispersion statistics used in the analysis. Let the horizontal position at time $t$ after release of drifter $m$ be given by $\mathbf{X}_{m}(t)=X_{m}(t) \hat{\mathbf{i}}+Y_{m}(t) \hat{\mathbf{j}}$, where $X_{m}\left(Y_{m}\right)$ are the easting (northing) of the drifter. The initial position of the drifter is $\mathbf{X}_{m, 0} \equiv \mathbf{X}_{m}(t=0)$. The vector between two drifters $m$ and $n$ is $\mathbf{r}_{m n}(t)=\mathbf{X}_{m}(t)-\mathbf{X}_{n}(t)$ with the initial separation vector given by $\mathbf{r}_{m n, 0}=\mathbf{X}_{m, 0}-\mathbf{X}_{n, 0}$. The squared separation for an individual drifter pair is given by

$$
r_{m n}^{2} \equiv\left\|\mathbf{r}_{m n}\right\|^{2}=\left\|\mathbf{X}_{m}-\mathbf{X}_{n}\right\|^{2} .
$$

For drifter $m$, the position relative to the initial position, the drifter displacement, is indicated by a superscripted prime $\mathbf{X}_{m}(t)=\mathbf{X}_{m}(t)-\mathbf{X}_{m, 0}$. The difference in displacements between drifter $m$ and $n$ is considered the perturbation separation $\mathbf{r}_{m n}^{\prime}(t)=\mathbf{r}_{m n}(t)-\mathbf{r}_{m n, 0}$ with squared distance

$$
r_{m n}^{\prime 2} \equiv\left\|\mathbf{r}_{m n}^{\prime}\right\|^{2}=\left\|\mathbf{r}_{m n}-\mathbf{r}_{m n, 0}\right\|^{2}=\left\|\mathbf{X}_{m}^{\prime}-\mathbf{X}_{n}^{\prime}\right\|^{2}
$$

Separation magnitudes and separation perturbation magnitudes are related through the law of cosines

$$
r_{m n}^{2}=r_{m n}^{\prime 2}+r_{m n, 0}^{2}+2 r_{m n, 0} r_{m n}^{\prime} \cos \theta_{m n}
$$

where $\theta_{m n}$ is the angle between $\mathbf{r}_{m n, 0}$ and $\mathbf{r}_{m n}^{\prime}(t)$. Relative, or two-particle dispersion is described by the statistics of $r_{m n}^{2}$ and $r_{m n}^{\prime 2}$.

The statistics of $r_{m n}$ and $r_{m n}^{\prime}$ over drifter pairs for a given initial separation $r_{0}$ are now defined. The dispersion $D_{r}^{2}(t)$ is mean squared separation

$$
D_{r}^{2}\left(t, r_{0}\right)=\left\langle r_{m n}^{2}\left(t \mid r_{0}\right)\right\rangle
$$

where the averaging $\langle\cdot\rangle$ is over all drifter pair separations $r_{m n}^{2}(t)$ with initial separation $r_{0}$. The number of pairs with $r_{0}$ initial separation is time dependent and denoted $N_{p}=N_{p}\left(t, r_{0}\right)$. Similarly, the perturbation dispersion is

$$
D_{r^{\prime}}^{2}\left(t, r_{0}\right)=\left\langle r_{m n}^{\prime 2}\left(t \mid r_{0}\right)\right\rangle
$$

Expressing the last term in (7) as a dot product, the dispersion and perturbation dispersion are related via

$$
D_{r}^{2}\left(t, r_{0}\right)=D_{r^{\prime}}^{2}\left(t, r_{0}\right)+r_{0}^{2}+2\left\langle\mathbf{r}_{m n, 0} \cdot \mathbf{r}_{m n}^{\prime}\left(t \mid r_{0}\right)\right\rangle,
$$

where it is assumed that all drifter pairs have the same initial separation $r_{0}^{2}=\left\langle r_{m n, 0}^{2}\right\rangle$. The third term on the RHS of (10), here denoted

$$
\Phi\left(t, r_{0}\right) \equiv 2\left\langle\mathbf{r}_{m n, 0} \cdot \mathbf{r}_{m n}^{\prime}\left(t \mid r_{0}\right)\right\rangle,
$$

is zero if the initial separation vector and the perturbation vector are uncorrelated. This is typically assumed (Batchelor 1950) in homogeneous isotropic turbulence and when the averaging is over drifter pairs from many independent realizations. However, in a laboratory experiment, this term was nonzero and could not be neglected (Ouellette et al. 2006).

The total diffusivity $K_{r}$ measures the average squared separation rate and is defined as

$$
K_{r}\left(t, r_{0}\right)=\frac{1}{4} \frac{d}{d t} D_{r}^{2}\left(t, r_{0}\right)
$$

The factor of $1 / 4$ is used in (12) so that if each drifter in a pair is independent of the other, the two-particle diffusivity $K_{r}$ equals Taylor's single particle diffusivity (Taylor 1922). This occurs, for example, for drifters separated by a distance larger than the largest eddy length scale. The perturbation diffusivity is

$$
K_{r^{\prime}}\left(t, r_{0}\right)=\frac{1}{4} \frac{d}{d t} D_{r^{\prime}}^{2}\left(t, r_{0}\right) .
$$

Using (10), and assuming all drifters are initially separated by $r_{0}$, the diffusivity and perturbation diffusivity are related by

$$
K_{r}\left(t, r_{0}\right)=K_{r^{\prime}}\left(t, r_{0}\right)+\frac{1}{4} \frac{d}{d t} \Phi\left(t, r_{0}\right) .
$$

Although $d \Phi\left(t, r_{0}\right) / d t$ is typically assumed to be zero, it will be shown that this term is important to the dispersion investigated here similarly to laboratory dispersion (Ouellette et al. 2006).

The variability of the dispersion and diffusivities is calculated as standard deviations. For the dispersion $D_{r}^{2}$, it is denoted $\sigma_{D_{r}^{2}}$, and estimated as

$$
\sigma_{D_{r}^{2}}^{2}\left(t, r_{0}\right)=\left\langle\left[r_{m n}^{2}\left(t \mid r_{0}\right)-D_{r}^{2}\left(t, r_{0}\right)\right]^{2}\right\rangle .
$$

The standard deviation of $D_{r^{\prime}}^{2}$ is found and denoted similarly.

\section{b. The Batchelor regime}

The RO scaling for the dispersion (1) in inertial subrange turbulence only depends on the mean dissipation rate $\bar{\epsilon}$, i.e., $D_{r}^{2}=C_{R} \bar{\epsilon} t^{3}$ where $C_{R}$ is a nondimensional constant (e.g., Salazar and Collins 2009). Thus, RO scaling is independent of $r_{0}$ and only formally valid if $\Phi \ll D_{r^{\prime}}^{2}$ and for times $\left(t \gg t_{B}\right)$ where perturbation dispersion is much larger than the initial separation $D_{r^{\prime}}^{2} \gg r_{0}^{2}$. When these conditions are met, $D_{r}^{2}(t) \approx D_{r^{\prime}}^{2}(t)$. For inertial subrange $k^{-5 / 3}$ turbulence, Batchelor (1950) derived scaling for the perturbation dispersion $D_{r^{\prime}}^{2}\left(t, r_{0}\right)=$ $\mathscr{C b}^{2}\left(r_{0}\right) t^{2}$, Eq. (3), for short times in which the dispersion depends on $r_{0}$. The time scale separating RO (1) and Batchelor scaling (3) is the Batchelor time $t_{B}$ (e.g., Salazar and Collins 2009), here defined as

$$
t_{B}\left(r_{0}\right)=\frac{1}{4} \frac{r_{0}}{\mathscr{U}\left(r_{0}\right)},
$$


where $\mathscr{C}\left(r_{0}\right)$ is the Batchelor velocity. According to inertial subrange theory, the transition from Batchelor (3) to RO dispersion (1) should be direct, i.e., the dispersion $D_{r^{\prime}}^{2} \sim t^{\gamma}$ ought to increase directly from $\gamma=2$ to $\gamma=3$. However, laboratory experiments indicated that the dispersion is weaker than Batchelor $(\gamma<2)$ as the dispersion transitions out of the Batchelor regime (Ouellette et al. 2006). Although, the Batchelor regime was originally derived for $k^{-5 / 3}$ inertial subrange turbulence, for $t \ll t_{B}$ Batchelor scaling $D_{r^{\prime}}^{2} \sim t^{2}$ (3) will be found for initial times for any velocity field with spectra $E \sim k^{-\beta}$ as long as $\beta<3$.

\section{c. Structure function definitions}

Here the separation $r$ dependent velocity structure function $S(r)$ is used to examine the spatial structure of the flow. Structure functions are fundamentally Eulerian statistics but can be estimated from drifters (e.g., Poje et al. 2017), although the results may be biased relative to $S(r)$ calculated from Eulerian data as divergent motions may preferentially place drifters in convergence zones (Pearson et al. 2019). We calculate $S(r)$ from drifter pair trajectories using (5). For $S(r)$, the averaging $\langle\cdot\rangle$ in (5) is over all drifter pair velocity increments $\delta \mathbf{u}_{m n}=\mathbf{u}_{m}\left(\mathbf{r}_{m}\right)-\mathbf{u}_{n}\left(\mathbf{r}_{n}\right)$ separated by $r=\left\|\mathbf{r}_{m n}\right\|$. Thus, the averaging for structure functions differs from the averaging for dispersion statistics. Unlike $D^{2}\left(t, r_{0}\right)$ and $K\left(t, r_{0}\right)$, where averaging is done over pairs of drifters at time $t$ separated initially by $r_{0}$, structure function $S(r)$ averaging is over drifter pairs and times for which the drifters are separated by $r$.

The total structure function is related to the $1 \mathrm{D}$ spacedlagged velocity correlation function $\rho(r)=\langle u(x+r) u(x)\rangle / \sigma_{u}^{2}$, where $\sigma_{u}^{2}$ is the velocity variance. Assuming isotropy and homogeneity

$$
S(r)=4 \sigma_{u}^{2}[1-\rho(r)] .
$$

When properly normalized, the structure function $S(r)$ is related to the wavenumber spectra $E(k)$ by Fourier transform as the space-lagged correlation function and $E(k)$ are Fourier transform pairs (Babiano et al. 1985). Thus, energy spectra, structure functions, and dispersion are linked via

$$
\begin{aligned}
E(k) & \sim k^{-\beta}, \\
S(r) & \sim r^{\beta-1}, \\
D_{r}^{2} & \sim t^{4 /(3-\beta)}, \\
K_{r} & \sim D_{r}^{(\beta+1) / 2} .
\end{aligned}
$$

These relationships (17) are valid for $\beta<3$ wavenumber spectra, $\Phi \ll D_{r^{\prime}}^{2}$, and for times $t \gg t_{B}$ corresponding to $D_{r^{\prime}}^{2} \gg r_{0}^{2}$ such that $D_{r^{\prime}}^{2} \approx D_{r}^{2}$ (e.g., Foussard et al. 2017). At $t \ll t_{B}$, the perturbation dispersion follows a Batchelor scaling $D_{r}^{\prime 2}=S\left(r_{0}\right) t^{2}$ for all $\beta<3$ with structure function $S\left(r_{0}\right) \sim r_{0}^{\beta-1}$ (17b). For $\beta=3$ in the $2 \mathrm{D}$ turbulent enstrophy cascade, $S \sim r^{2}$ and the dispersion for all $t$ is given by $D_{r}^{2} \sim \exp (t)$ and $K_{r} \sim D_{r}^{2}(\operatorname{Lin} 1972)$. Thus, a Batchelor regime does not exist for a $k^{-3}$ spectra.

\section{Methods}

\section{a. Lagrangian data}

Drifter releases were performed during September and October of 2107 as part of the Office of Naval Researchfunded Inner Shelf experiment conducted near Pt. Sal, CA (Kumar et al. 2021; Spydell et al. 2019). Unlike many previous studies (e.g., Ollitrault et al. 2005; Koszalka et al. 2009; Lumpkin and Elipot 2010; Beron-Vera and LaCasce 2016), but similar to Ohlmann et al. (2012) where there were $\approx 12$ drifter release realizations, drifters were repeatedly released in the same geographic area increasing the number of independent drifter release realizations. Coastal Ocean Dynamics Experiment (CODE) drifter bodies (Davis 1985) were equipped with SPOT Trace GPS receivers (Subbaraya et al. 2016; Novelli et al. 2017) nominally sampling every $2.5 \mathrm{~min}$. SPOTs have been used in other oceanographic drifter studies (Beron-Vera and LaCasce 2016; Pearson et al. 2019) and methods to reduce their errors developed (Yaremchuk and Coelho 2015). Consistent with previously reported SPOT position errors between 2 and $10 \mathrm{~m}$ (Yaremchuk and Coelho 2015; Novelli et al. 2017), we estimate SPOT errors to be $\approx 4 \mathrm{~m}$ based on comparing SPOTs that were codeployed with higher-accuracy GPSs on some drifters. Drifters followed the mean surface horizontal flow between approximately 0.3 to $1.2 \mathrm{~m}$ below the surface. The water following properties of CODE drifters is well established (Poulain 1999; Novelli et al. 2017). Wind-induced drifter slips $\left(<0.01 \mathrm{~m} \mathrm{~s}^{-1}, 0.1 \%\right.$ of wind speed; Poulain 1999; Poulain and Gerin 2019) were small compared to the currents $\left(\approx 0.15 \mathrm{~m} \mathrm{~s}^{-1}\right)$ as wind speeds during drifter releases were much less than the maximum midafternoon wind speed $10 \mathrm{~m} \mathrm{~s}^{-1}$ recorded on two of the days.

Drifters were released in 10-40-m water depths (Fig. 1a). Here, 12 drifter releases are analyzed: 8 releases were off of the rocky Pt Sal headland (see Fig. 1b for an example release) and 4 releases were off the long sandy beach area called Oceano (dots in Fig. 1a are initial drifter positions for each release colored by latitude, blues to the south, reds to the north). Trajectories varied from 3 to $23 \mathrm{~h}$ long depending on the release leading to the median length of drifter pair trajectories ranging from 3.2 to $22.9 \mathrm{~h}$ (Table 1 ). The relatively long pair trajectories from release 4 and 9 are due to some drifters being left out overnight. The drifter deployment pattern, multiple groups of 9 drifters arranged in a plus pattern (blue dots Fig. 1b), consists of various initial separations $r_{0}$ from 100 to $2000 \mathrm{~m}$. For each release, all drifters were deployed within approximately $35 \mathrm{~min}$ - the mean over the 12 releases of the time it took to deploy the drifters for each release. As the deployments were quickly performed, time $t$ for drifter statistics, such as $D_{r}^{2}(t)$, can be considered the time since deployment (column 2 in Table 1) in UTC.

SPOT GPS data contain gaps (e.g., Yaremchuk and Coelho $2015)$ with the time between fixes $\delta \tau \geq 2.5 \mathrm{~min}$. For the trajectories used here, $91 \%$ of all $\delta \tau$ is $\leq 5 \mathrm{~min}$ with the mean time between fixes $\overline{\delta \tau}=192 \mathrm{~s}$ (rather than $150 \mathrm{~s}$ as prescribed). Large gaps occurred more often during calm conditions because the SPOT Trace GPS units used here required accelerations (from surface gravity waves) to continuously transmit. Trajectories with gaps larger than $\delta \tau_{m x}=45 \mathrm{~min}$ 

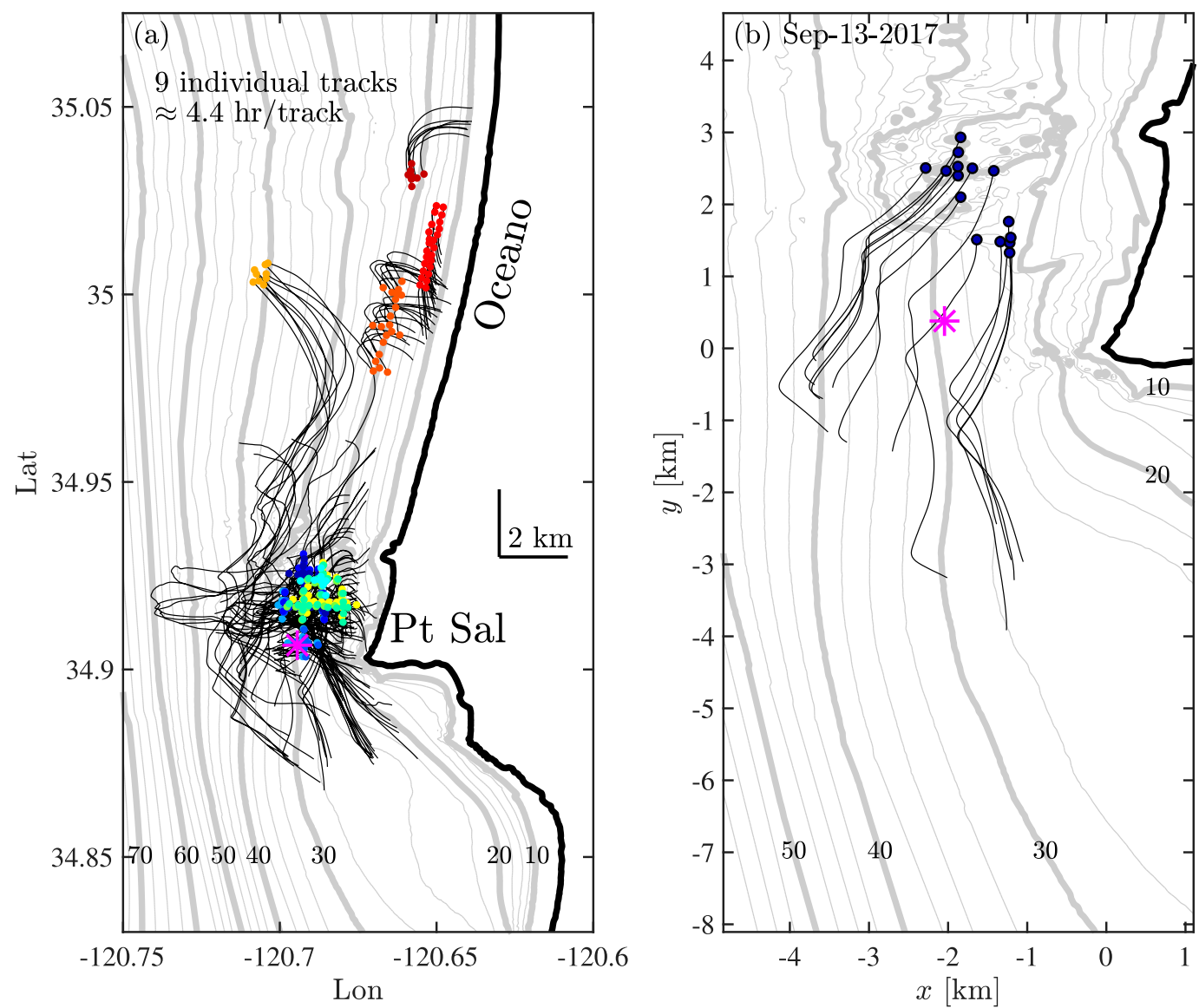

FIG. 1. (a) The Pt. Sal drifter trajectories. There are 12 releases of 8-26 drifters. Dots are the initial positions colored according to the latitude of the release: blues to the south, reds to the north. (b) An example realization showing trajectories for drifters released on 13 Sep 2017 in coordinates centered on the tip of Pt. Sal. Magenta asterisks indicate the location of the ADCP and temperature mooring used in the analysis. Bathymetry is contoured in gray: thick contours are separated by $10 \mathrm{~m}$, and thin contours are separated by $2.5 \mathrm{~m}$ (starting at $10 \mathrm{~m}$ ).

are not included in the analysis. For each release, the number of trajectories $N$ meeting this requirement (all $\delta \tau<$ $\left.\delta \tau_{m x}\right)$, and the number of drifter pairs $N_{p}$, are shown in Table 1. The number of pairs $N_{p}$ sometimes differs from $N(N-1) / 2$, the number of pairs given $N$ drifters, because, infrequently, two trajectories from the same release do not overlap in time. The results reported here do not depend on the gap criterion $\delta \tau_{m x}$ and nearly identical results are obtained if the requirement is loosened (e.g., $\delta \tau_{m x}=1 \mathrm{~h}$ ) or tightened (e.g., $\delta \tau_{m x}=15 \mathrm{~min}$ ).

Drifter trajectories are processed as follows. 1) GPS latitude-longitude fixes, sampled at $2.5 \mathrm{~min}$, are projected onto the local UTM plane. 2) Easting and northing drifter positions with gaps are differenced to obtain velocities. 3) Velocities are then linearly interpolated to times separated by $2.5 \mathrm{~min}$ filling any gaps. 4) Velocities are integrated to obtain positions with the constant determined such that the mean square difference between the original trajectory and the interpolated trajectory is minimized. 5) Position spikes are removed by linearly interpolating positions for which acceleration (velocity differences) magnitudes are $>0.0387 \mathrm{~m} \mathrm{~s}^{-2}$. This acceleration magnitude removes all obvious outlier positions. Only $0.13 \%$ of all positions required despiking. Velocities are then recomputed from despiked positions. 6) A 5 min ( 3 point) moving boxcar average is then applied to positions and velocities resulting in the drifter positions $\mathbf{X}(t)$ and velocities $\mathbf{U}(t)$ analyzed here. Assuming independent (every $2.5 \mathrm{~min}$ ) position errors of $4 \mathrm{~m}, 5$-min averaged positions $\mathbf{X}(t)$ have approximately $2.3 \mathrm{~m}$ errors and 5 -min averaged velocity $\mathbf{U}(t)$ errors are approximately $0.015 \mathrm{~m} \mathrm{~s}^{-1}$.

Results are presented for two different averages. First, experiment averaged (EA) drifter statistics are presented for which the averaging $\langle\cdot\rangle$ in (8), (9), and (15) is over all possible drifter pairs from the entire experiment, i.e., averaging over all 12 drifter releases. Second, single release (SR) averaged statistics are presented for which the averaging is only over drifter pairs for a particular release. Thus, SR statistics are based on averages over fewer drifter pairs than EA statistics. For EA statistics, there are a total of 2187 drifter pairs $\left(N_{p}\right.$ in last row of Table 1). For the entire experiment, the majority of initial 
TABLE 1. Pt. Sal drifter release information. The starting time (column 2) of each release, the number of drifters (column 3), the total number of drifter pairs (column 4), initial separation information (columns 5-7), and pair trajectory length $T_{p}$ (columns 8-10) information.

\begin{tabular}{|c|c|c|c|c|c|c|c|c|c|}
\hline Release & $\begin{array}{c}\text { Date (of 2017) and } \\
\text { hour (UTC) of release }\end{array}$ & $N$ & $N_{p}$ & $\begin{array}{c}\operatorname{Min} \\
r_{0}(\mathrm{~m})\end{array}$ & $\begin{array}{c}\text { Median } \\
r_{0}(\mathrm{~m})\end{array}$ & $\begin{array}{c}\operatorname{Max} \\
r_{0}(\mathrm{~m})\end{array}$ & $\begin{array}{c}\text { Min } \\
T_{p}(\mathrm{~h})\end{array}$ & $\begin{array}{c}\text { Median } \\
T_{p}(\mathrm{~h})\end{array}$ & $\begin{array}{l}\operatorname{Max} \\
T_{p}(\mathrm{~h})\end{array}$ \\
\hline 1 & 10 Sep, 1520 & 24 & 276 & 110 & 1100 & 2100 & 3.5 & 3.9 & 5.1 \\
\hline 2 & 11 Sep, 1610 & 23 & 253 & 100 & 1100 & 2100 & 3.8 & 4.3 & 5.8 \\
\hline 3 & 12 Sep, 1500 & 22 & 231 & 60 & 1000 & 2000 & 4.3 & 5.3 & 6.9 \\
\hline 4 & 12 Sep, 2230 & 9 & 36 & 70 & 300 & 700 & 22.7 & 22.9 & 23 \\
\hline 5 & 13 Sep, 1440 & 15 & 105 & 10 & 600 & 1400 & 4.0 & 4.8 & 5.5 \\
\hline 6 & 14 Sep, 1430 & 8 & 21 & 80 & 300 & 700 & 4.5 & 4.6 & 4.9 \\
\hline 7 & 16 Sep, 1450 & 21 & 210 & 90 & 1200 & 3100 & 3.9 & 4.4 & 6.3 \\
\hline 8 & 17 Sep, 1500 & 22 & 231 & 120 & 900 & 2200 & 3.3 & 3.7 & 4.8 \\
\hline 9 & 09 Oct, 1550 & 19 & 171 & 140 & 700 & 1500 & 3.9 & 4.9 & 22 \\
\hline 10 & 10 Oct, 1500 & 26 & 325 & 60 & 900 & 2000 & 5.1 & 5.5 & 6.2 \\
\hline 11 & 13 Oct, 1450 & 25 & 300 & 120 & 800 & 2500 & 2.9 & 3.2 & 3.8 \\
\hline 12 & 14 Oct, 1500 & 8 & 28 & 130 & 400 & 800 & 4.2 & 4.5 & 4.7 \\
\hline All & & 222 & 2187 & & 900 & & & 4.4 & \\
\hline
\end{tabular}

separations $r_{0}$ are $\leq 1500 \mathrm{~m}$ (Fig. 2). Initial separations $r_{0}$ are binned every $250 \mathrm{~m}$ from 250 to $3000 \mathrm{~m}$ (bin centers) with $>200$ drifter pairs for $r_{0} \leq 1500 \mathrm{~m}$, whereas there are fewer drifter pairs $\left(N_{p} \leq 100\right)$ for $r_{0} \geq 1750 \mathrm{~m}$. The mean $r_{0}$ within each bin, for $250 \leq r_{0} \leq 1500 \mathrm{~m}$, is very close to the bin center (see red plus signs in Fig. 2). For this reason, and because there are few pairs for $r_{0} \geq 1750 \mathrm{~m}$, only results for $250 \leq r_{0} \leq 1500 \mathrm{~m}$ are presented for which there are a total of 1998 drifter pairs. The number of drifter pairs used for experiment averaged statistics at each $r_{0}$ is larger than some previous studies (e.g., Koszalka et al. 2009; Ohlmann et al. 2012) with similar $r_{0}$, thus, the experiment averaged statistics reported here are robust relative to previous estimates for $r_{0} \leq 1500 \mathrm{~m}$. Statistics depend on time $t$ where $t=0$ is the first time for each drifter pair trajectory, i.e., the time of the first GPS fix for which both drifters are in the water. Relative dispersion statistics are not affected by the time gap between drifter deployments as statistics are only a function of time $t$ since both drifters are deployed. For experiment averaged statistics time $t$ does not correspond to a UTC time whereas for single release statistics, assuming drifters were rapidly deployed, $t$ is the time in UTC since deployment.

\section{b. Eulerian data}

For the two months of the Pt. Sal experiment, 46 collocated upward-looking ADCPs and temperature moorings were deployed. Velocity and temperatures averaged to $10 \mathrm{~min}$ resolution from one mooring deployed in $30 \mathrm{~m}$ water depth close to the drifter releases [pink asterisk at $\approx(-2,0) \mathrm{km}$ in Figs. 1a,b] are used in the analysis. McSweeney et al. (2020) provides a thorough description of the moorings.

\section{Results}

Here we examine two-particle dispersion statistics. In particular, we examine the effects of drifter initial separation, the difference between perturbation and total separation statistics, and the relationship between dispersion and structure functions. Results are presented first for experiment averaged (EA) dispersion and then for single release (SR) dispersion.

\section{a. Experiment averaged two-particle statistics}

Experiment averaged (EA) dispersion statistics are calculated as long as there are a sufficient number of drifter pairs. The number of drifter pairs $N_{p}$ depends on the initial separation $r_{0}$ and time $t$ (Fig. 3a). The number of drifter pairs is constant in time for $t<10^{4} \mathrm{~s}$ and equal to the initial number of pairs (Fig. 2) before rapidly dropping as drifters were picked up. For each $r_{0}$, the EA dispersion statistics are displayed only for $N_{p}(t)>200$ (gray line, Fig. 3a) which is effectively $t<10^{4} \mathrm{~s}$. Including fewer drifter pairs yields noisy statistics for these times.

The experiment averaged (EA) perturbation dispersion scales like $D_{r^{\prime}}^{2}\left(t, r_{0}\right)=\mathscr{U}^{2}\left(r_{0}\right) t^{2}$ for $r_{0} \geq 500 \mathrm{~m}$ (colored curves in Fig. 3b) for approximately $t<5000 \mathrm{~s}$. For the smallest $r_{0}=$ $250 \mathrm{~m}$, the growth is slightly slower than $t^{2}\left(\approx t^{1.85}\right)$. The slightly slower than $\sim t^{2}$ perturbation dispersion growth for $r_{0}=250 \mathrm{~m}$ (and to a lesser extent $r_{0}=500 \mathrm{~m}$ ) could be due to GPS position errors that are inversely correlated with drifter separation. Such GPS correlated GPS position errors have been observed for another type of GPS receiver (Spydell et al. 2019). Such correlated GPS position error may cause the estimated $D_{r^{\prime}}^{2}\left(t, r_{0}\right)$ to grow slower than $t^{2}$. The ballistic growth $\mathscr{C b}^{2}\left(r_{0}\right)$ increases with $r_{0}$ (stacking of colored curves in Fig. $3 b$ ).

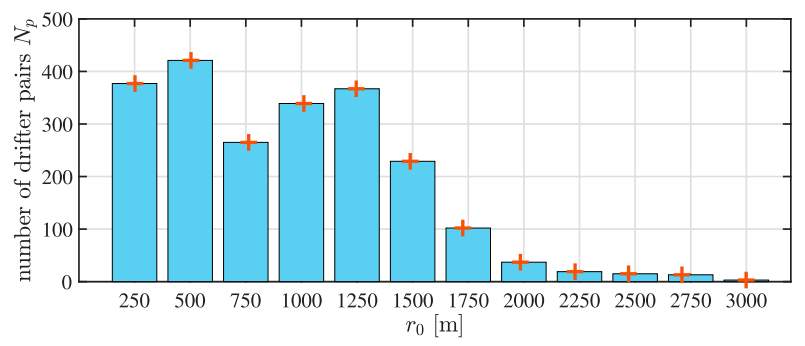

FIG. 2. Distribution of initial drifter separations $r_{0}$ used for experiment averaged statistics. The actual bin centers are indicated by red plus signs and do not deviate much from multiples of $250 \mathrm{~m}$, especially for $r_{0} \leq 1500 \mathrm{~m}$. 

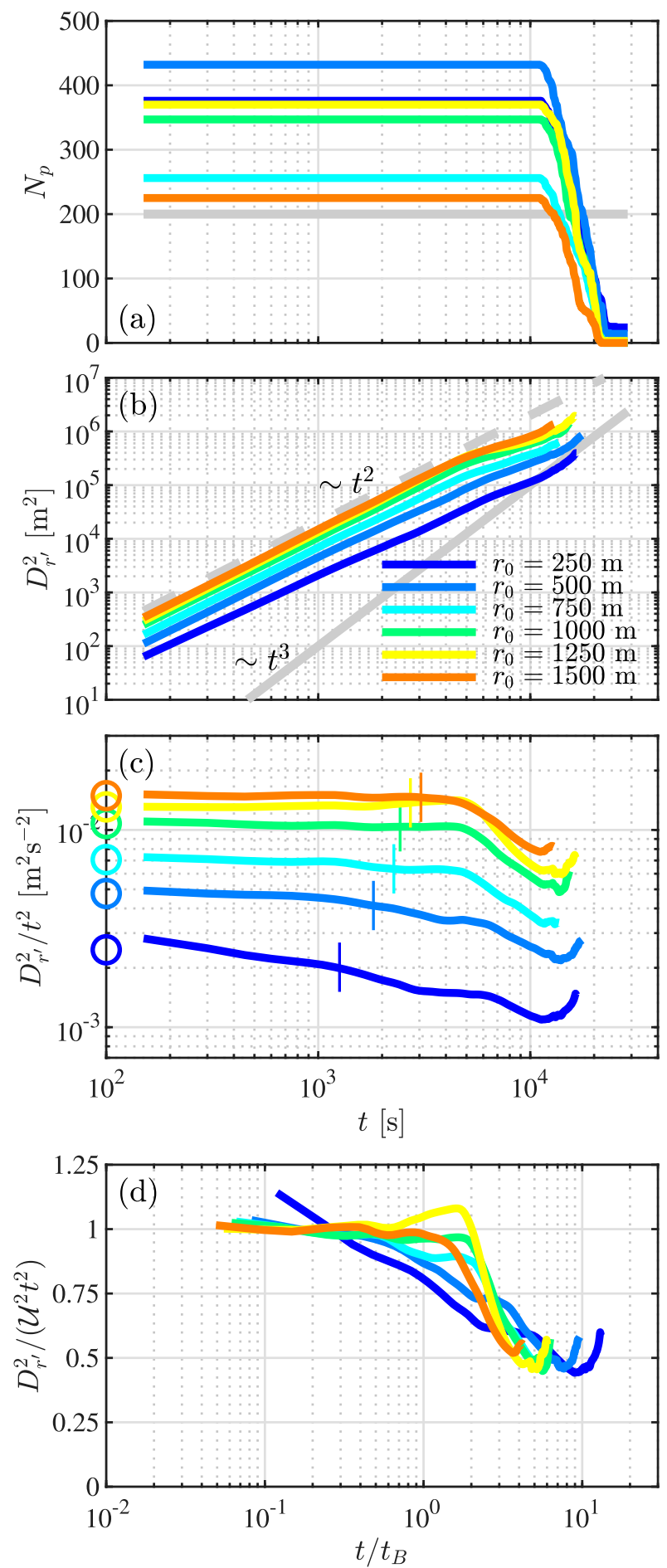

FIG. 3. (a)-(c) Experiment averaged (EA) two-particle statistics vs time $t$. (a) The number of drifter pairs $\left(N_{p}\right)$ vs time $t$ for initial separations $250 \geq r_{0} \geq 1500 \mathrm{~m}$ (colors). Minimum number of pairs $N_{p}^{(\min )}=200$ for analysis is indicated by the horizontal gray line. (b) The EA perturbation dispersion $D_{r^{\prime}}^{2}$ (9) vs time $t$. (c) The compensated EA perturbation dispersion $D_{r^{\prime}}^{2} / t^{2}$ vs time $t$. (d) The scaled EA perturbation dispersion $D_{r^{\prime}}^{2} /\left(\mathscr{b}^{2} t^{2}\right)$ vs scaled time $t / t_{B}$. The dispersion curves are only shown for times with $N_{p}(t)>N_{p}^{(\min )}$.
Thus, the initial EA perturbation dispersion is consistent with a Batchelor regime as $D_{r^{\prime}}^{2}=\mathscr{U}^{2}\left(r_{0}\right) t^{2}(3)$ and $\mathscr{C}^{2}\left(r_{0}\right)$ increases with $r_{0}$ (Salazar and Collins 2009). Although some oceanographic studies have found RO scaling over multiple decades (e.g., Poje et al. 2017), the durations of these drifter releases were too short to observe classic RO scaling $D_{r^{\prime}}^{2} \sim t^{3}$ (solid gray line in Fig. 3b) for which the different $r_{0}$ curves in Fig. $3 b$ would collapse to a single curve. Thus, the focus here is on Batchelor scaling rather than RO scaling. However, the steepening of the $D_{r^{\prime}}^{2}$ curves for long times $\left(t>10^{4} \mathrm{~s}\right.$ Fig. 3b) suggests that the dispersion maybe transitioning from $t^{2}$ to $t^{3}$ growth.

The EA perturbation dispersion compensated by $t^{2}$ clearly shows a Batchelor scaling for $r_{0} \geq 500 \mathrm{~m}$ (Fig. 3c). Assuming Batchelor scaling for the EA perturbation dispersion, the Batchelor velocity $\mathscr{C}\left(r_{0}\right)$ is estimated here from drifter data using

$$
\mathscr{U}^{2}\left(r_{0}\right)=\overline{D_{r^{\prime}}^{2}\left(t, r_{0}\right) / t^{2}}
$$

where the overline represents a time mean from 150 to $600 \mathrm{~s}$. Thus, $\mathscr{C}$ is estimated using only the first $600 \mathrm{~s}$ of drifter pair data. Consistent with theoretical expectations (Salazar and Collins 2009), squared Batchelor velocities increase with $r_{0}$ (circles placed at $t=10^{2} \mathrm{~s}$ in Fig. 3c) from $0.0026 \mathrm{~m}^{2} \mathrm{~s}^{-2}$ at $r_{0}=$ $250 \mathrm{~m}$ to $0.0149 \mathrm{~m}^{2} \mathrm{~s}^{-2}$ at $r_{0}=1500 \mathrm{~m}$. Also consistent with theoretical expectations, comparing colored curves in Fig. $3 \mathrm{c}$ shows that the duration of Batchelor scaling increases with $r_{0}$ as cooler colored curves depart from a constant sooner than the warmer colored curves (Ouellette et al. 2006). Thus, the Batchelor time $t_{B}\left(r_{0}\right)=0.25 r_{0} \mathscr{b ^ { - 1 }}$ (16) increases with $r_{0}$ (thin vertical lines in Fig. 3c). Except for the smallest initial separation $r_{0}=250 \mathrm{~m}$, the Batchelor time $t_{B}$ is consistent with $D_{r^{\prime}}^{2}$ changing by approximately $15 \%$ for all $r_{0}$. The decreasing in time $t$ compensated EA perturbation dispersion $D_{r^{\prime}}^{2}\left(t, r_{0}\right) / t^{2}$, beginning at $t=200 \mathrm{~s}$ for $r_{0}=250 \mathrm{~m}$ and $t=4000 \mathrm{~s}$ for $r_{0}=$ $1500 \mathrm{~m}$ (Fig. 3c), indicates that the dispersion is weaker than Batchelor $\left(t^{\gamma}\right.$ with $\left.\gamma<2\right)$ when transitioning out of Batchelor scaling.

Scaling EA $D_{r^{\prime}}^{2}$ for each $r_{0}$ by $2 b^{2}\left(r_{0}\right) t^{2}$, and time $t$ by the Batchelor time $t_{B}\left(r_{0}\right)$, collapses the perturbation dispersion fairly well overall (Fig. 3d). The collapse is very good for $750 \leq$ $r_{0} \leq 1500 \mathrm{~m}$ (cyan to orange curves) for which the scaled EA perturbation dispersions are all generally similar to each whereas for $r_{0}=250 \mathrm{~m}$ and $500 \mathrm{~m}$ the collapse is not as good. For $r_{0} \geq 750 \mathrm{~m}, t_{B}$ well predicts the time when $D_{r^{\prime}}^{2} /\left(\mathscr{b ^ { 2 }} t^{2}\right)$ begins to rapidly drop (when $t / t_{B} \approx 2$. Thus, for the $r_{0} \geq 750 \mathrm{~m}$ that show the best Batchelor scaling $\left(D_{r^{\prime}}^{2} \sim t^{2}\right.$, e.g., Fig. $\left.3 c\right), t_{B}$ indicates well the duration of $D_{r^{\prime}}^{2} \sim t^{2}$ growth, while for the $r_{0} \leq 500 \mathrm{~m}$ that show weaker than $\sim t^{2}$ growth, $t_{B}$ does not correspond to the duration of the initial growth. The scaled

\section{$\longleftarrow$}

In (b), the dashed gray line is $t^{2}$ and the solid gray line is $\sim t^{3}$. In (c), for each $r_{0}$, circles indicate squared Batchelor velocities $\mathscr{C}^{2}\left(r_{0}\right)(18)$ and tick marks indicate the Batchelor time $t_{B}(16)$. 
dispersions for $r_{0}=250 \mathrm{~m}$ and $500 \mathrm{~m}$ (blue curves) drop less rapidly than for $r_{0} \geq 750 \mathrm{~m}$. For all $r_{0}$, the departure from Batchelor scaling, and subsequent $D_{r^{\prime}}^{2}$ growth slower than $t^{2}$, results in perturbation dispersions for long times that are approximately $50 \%$ of the dispersion that would result if Batchelor scaling $D_{r^{\prime}}^{2}=\mathscr{b ^ { 2 }} t^{2}$ remained valid for all $t$, i.e., for all curves, eventually $D_{r^{\prime}}^{2} /\left(\mathscr{b ^ { 2 }} t^{2}\right) \approx 0.5$ for $t / t_{B}>1$ (Fig. 3d). This also indicates that the dispersion weakens when transitioning out of the Batchelor regime similar to laboratory experiments (Ouellette et al. 2006).

In contrast to EA $D_{r^{\prime}}^{2}(t)$, the EA total dispersion $D_{r}^{2}(t)(8)$ shows no evidence of a power law scaling for $t<10^{4} \mathrm{~s}$ (Fig. 4a). For each $r_{0}$, EA $D_{r}^{2}(t)$ increases in time from a constant. Relative to the initial $D_{r}^{2}$, the increase in time from the constant $r_{0}^{2}$ is largest ( $\geqq 8$ times) for the smallest $r_{0}=$ $250 \mathrm{~m}$ and smallest ( $\geqq 2$ times) for the largest $r_{0}=1500 \mathrm{~m}$. For each $r_{0}$, the total dispersion $D_{r}^{2}\left(t, r_{0}\right)(8)$ is similar to $r_{0}^{2}+D_{r^{\prime}}^{2}\left(t, r_{0}\right)$ (cf. colored and thin black curves in Fig. 4a). The deviation $D_{r^{\prime}}^{2}\left(t, r_{0}\right)-\left[r_{0}^{2}+D_{r^{\prime}}^{2}\left(t, r_{0}\right)\right]$ is quantified by $\Phi\left(t, r_{0}\right)$, see (10) and (11), hence $\Phi$ is estimated as $\Phi\left(t, r_{0}\right)=$ $D_{r}^{2}\left(t, r_{0}\right)-r_{0}^{2}-D_{r^{\prime}}^{2}\left(t, r_{0}\right)$ (colored curves in Fig. 3b). The deviation $\Phi(10)$ is due to the correlation between the initial separation and perturbation separation $\left\langle\mathbf{r}_{0} \cdot \mathbf{r}^{\prime}(t)\right\rangle$ directly calculated using (11) (thin black curves in Fig. 4b). In accordance with the theory, directly calculating $\Phi$ using (11) is identical to calculating $\Phi$ from dispersion residuals (thin black and colored curves are indistinguishable in Fig. 4b). For all $r_{0}$, initially $\Phi$ is larger than $D_{r^{\prime}}^{2}$ (cf. Figs. $3 \mathrm{~b}$ and $4 \mathrm{~b}$ ) but for larger times $D_{r^{\prime}}^{2}(t)>\Phi(t)$ due to faster growth of $D_{r^{\prime}}^{2}(t) \sim t^{2}$ compared to $\Phi \sim t$. The time $t_{\Phi}\left(r_{0}\right)$ when $\Phi\left(t, r_{0}\right)=D_{r^{\prime}}^{2}\left(t, r_{0}\right)$ generally increases with $r_{0}$ (circles in Fig. 4b). For $t<t_{\Phi}, \Phi$ initially contributes more to $D_{r}^{2}$ than does $D_{r^{\prime}}^{2}$ and vice versa. Thus, for $t<t_{\Phi}$ the quantity $D_{r}^{2}-r_{0}^{2}=D_{r^{\prime}}^{2}+\Phi$ differs considerably from $D_{r^{\prime}}^{2}$ and grows as $\sim t$ due to the $\Phi$ contribution. The total dispersion $D_{r^{\prime}}^{2}$ does not follow the alternative scaling law $D_{r^{\prime}}^{2}\left(t, r_{0}\right)=$ $\left[r_{0}+\mathscr{U}\left(r_{0}\right) t\right]^{2}$ as the cross term $2 r_{0} \mathscr{U}\left(r_{0}\right) t \neq \Phi$ due to $r_{0} \mathscr{\mathscr { C }}\left(r_{0}\right) t \neq$ $\left\langle\mathbf{r}_{0} \cdot \mathbf{r}^{\prime}\right\rangle$ (not shown). Physically, a $\Phi>0$ with $d \Phi / d t>0$ represents particles on average moving away from each other. For $\Phi \sim t$, particles are on average moving away from each other at a constant velocity.

The EA perturbation diffusivity $K_{r^{\prime}}\left(t, r_{0}\right)$ is calculated from $D_{r^{\prime}}^{2}\left(t, r_{0}\right)$ using (13). For approximately $t<4000 \mathrm{~s}, K_{r^{\prime}} \sim t$ (Fig. 5a) consistent with a Batchelor regime. In contrast, the total diffusivity $K_{r}\left(t, r_{0}\right)$ is considerably different (Fig. 5b) than $K_{r^{\prime}}\left(t, r_{0}\right)$. For $r_{0}=1250,1500 \mathrm{~m}, K_{r}(t)$ is nearly constant for all $t$ whereas for $r_{0}=250,500 \mathrm{~m}, K_{r}(t)$ increases more quickly in time. Specifically, $K_{r}(t)$ changes by a factor of $\approx 1.7$ times for $r_{0}=1500 \mathrm{~m}$ and by $\approx 12.5$ times for $r_{0}=250 \mathrm{~m}$. The difference between $K_{r^{\prime}}$ and $K_{r}$ is due to $d \Phi / d t$ where $0.25 d \Phi / d t=\left(K_{r}-K_{r^{\prime}}\right)(14)$. As $\Phi$ increases linearly or more

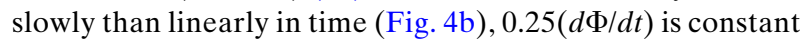
or slowly decreasing in time for all $r_{0}$ (Fig. $5 \mathrm{c}$ ). Owing to the different growth rates for $K_{r^{\prime}}(t)$ and $0.25(d \Phi / d t)$, for $t<t_{0}$, where $t_{0}$ is the time when $0.25(d \Phi / d t)=K_{r^{\prime}}(t)$ (circles in Fig. 5c), $0.25(d \Phi / d t)$ contributes more to $K_{r}(t)$ than the perturbation diffusivity $K_{r^{\prime}}$. For $t>t_{0}, d \Phi / d t$ contributes less to $K_{r}(t)$ than the perturbation diffusivity $K_{r^{\prime}}$. This transition
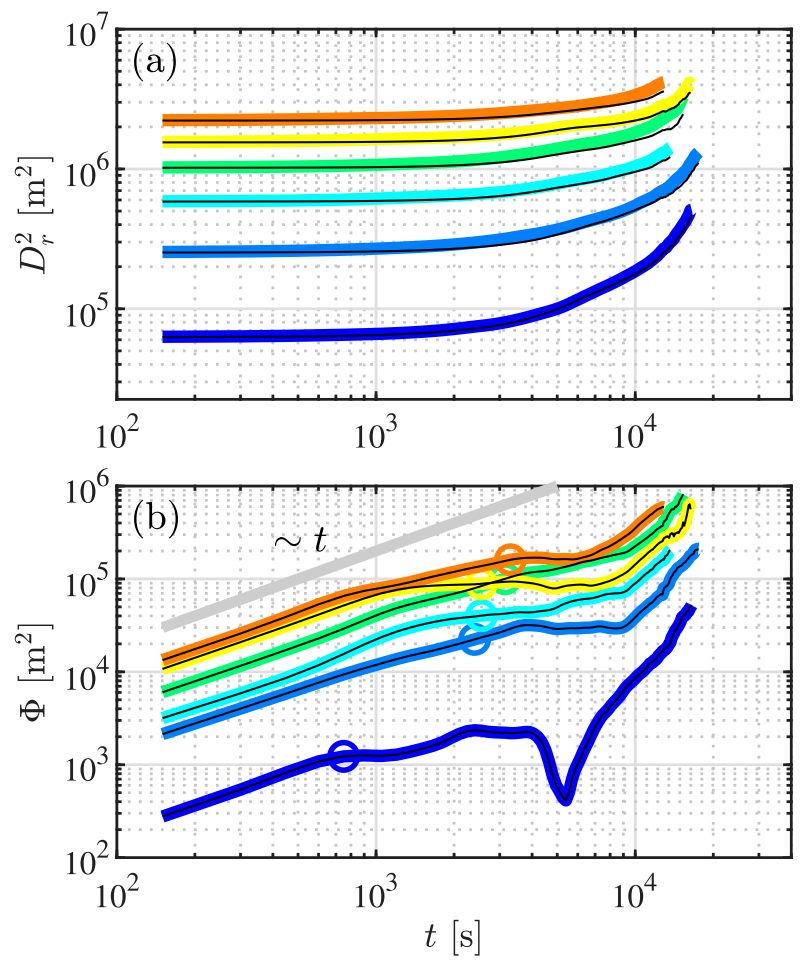

FIG. 4. (a) The experiment averaged dispersion $D_{r}^{2}(8)$ and (b) $\Phi$ (11) vs time $t$. In (a), thin black curves are $r_{0}^{2}+D_{r^{\prime}}^{2}(t)$. In (b), colored curves are $\Phi=D_{r^{\prime}}^{2}-D_{r^{\prime}}^{2}-r_{0}^{2}$, thin black curves are $\Phi$ $=2\left\langle\mathbf{r}_{0} \cdot \mathbf{r}^{\prime}\right\rangle$, and circles indicate the time $t_{\Phi}$ when $\Phi(t)=D_{r^{\prime}}^{2}(t)$. Colors correspond to initial separations $250 \leq r_{0} \leq 1500 \mathrm{~m}$ (see legend in Fig. 3). In (a) and (b), thin black curves are on top of colored curves for all $r_{0}$.

time $t_{0}$ generally increases with $r_{0}$ (circles in Fig. $5 \mathrm{c}$ ). The mean separation velocity $v_{r}$ is found from $\Phi$

$$
v_{r}=\frac{1}{2 r_{0}} \frac{d}{d t} \Phi\left(t, r_{0}\right)
$$

and initially $(t \approx 100 \mathrm{~s})$ increases with $r_{0}$ from about $0.004 \mathrm{~m} \mathrm{~s}^{-1}$ to $\approx 0.03 \mathrm{~m} \mathrm{~s}^{-1}$ (Fig. 5d). The initially constant $v_{r}$ for each $r_{0}$ results from $\Phi \sim t$ and indicates that particles on average are moving away from each other at a constant velocity that increases with initial particle separation. For all $r_{0}, v_{r}$ generally decreases in time until $t \approx 4000 \mathrm{~s}$ before generally increasing in time from 4000 to $10000 \mathrm{~s}$.

The dependence of the EA diffusivity $K_{r^{\prime}}$ on scale is now explored. Within the Batchelor regime, $K_{r^{\prime}} \sim\left(D_{r^{\prime}}^{2}\right)^{1 / 2}$ (Fig. 6a) since $D_{r^{\prime}}^{2} \sim t^{2}$ and $K_{r^{\prime}} \sim t$. The maximum length scale $\left(D_{r^{\prime}}^{2}\right)^{1 / 2}$ for which the scaling $K_{r^{\prime}} \sim\left(D_{r^{\prime}}^{2}\right)^{1 / 2}$ applies increases with $r_{0}$ consistent with the requirement that $D_{r^{\prime}}^{2}<r_{0}^{2}$ within the Batchelor regime. For instance, $K_{r^{\prime}}$ deviates from $K_{r^{\prime}} \sim\left(D_{r^{\prime}}^{2}\right)^{1 / 2}$ at smaller $\left(D_{r^{\prime}}^{2}\right)^{1 / 2}$ for $r_{0}=250 \mathrm{~m}$ than for $r_{0}=1500 \mathrm{~m}$ (cf. blue and orange curves in Fig. 6a). The $r_{0}$-dependent $K_{r^{\prime}} \sim D_{r^{\prime}}$ Batchelor scaling is contrasted with RO scaling for which $K_{r^{\prime}} \sim D_{r^{\prime}}^{4 / 3}$ independent of $r_{0}$ (thick solid gray line in Fig. 6a). However, the perturbation diffusivity $K_{r^{\prime}}$ appears to approach a $r_{0}$-independent 

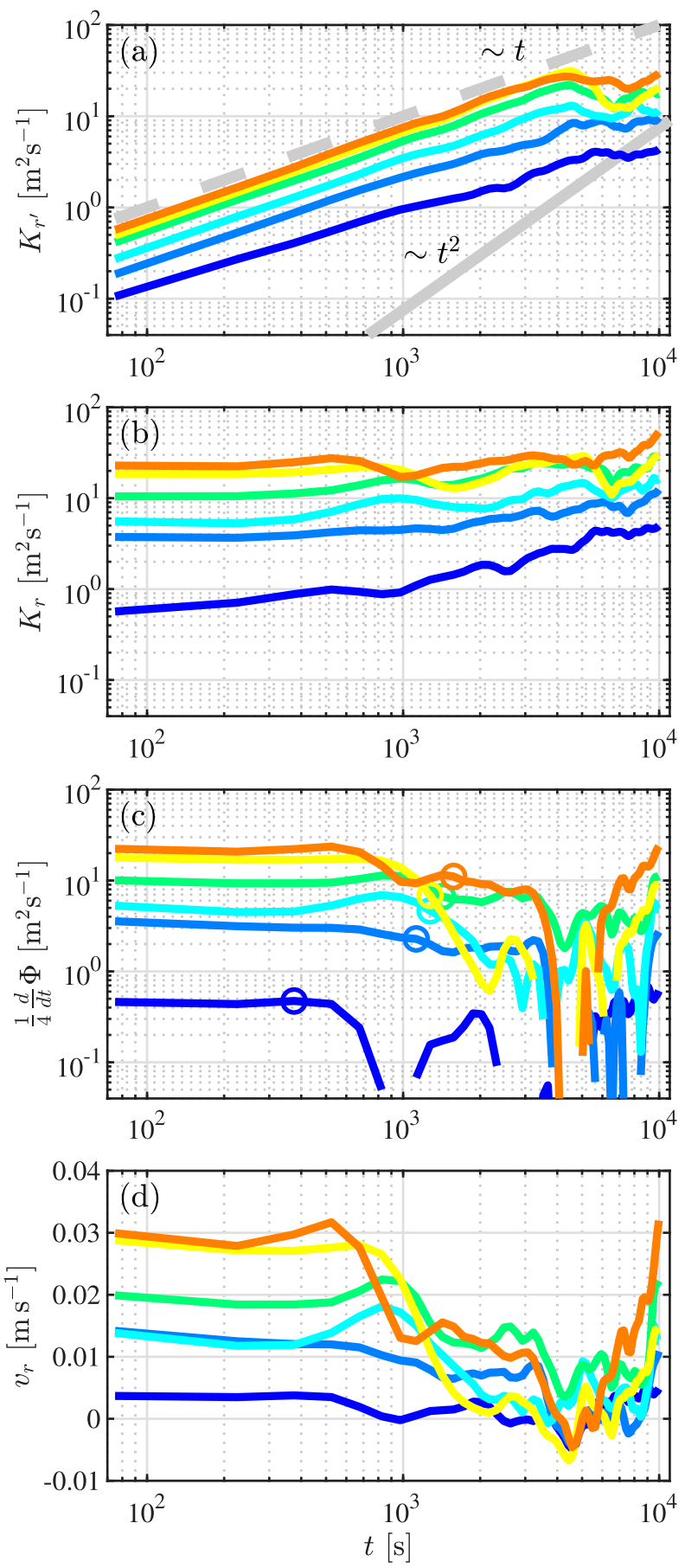

FIG. 5. (a) The experiment averaged (EA) perturbation diffusivity $K_{r^{\prime}}(13)$, (b) the EA total diffusivity $K_{r}(12)$, (c) the EA difference $1 / 4(d \Phi / d t)=K_{r}-K_{r^{\prime}}(14)$, and (d) the EA separation velocity $v_{r}(19)$ vs time $t$. In (a), the Batchelor scaling $\sim t$ (dashed gray) and the RO scaling $\sim t^{2}$ (solid gray) are shown. In (c), circles indicate the time when $K_{r^{\prime}}=1 / 4(d \Phi / d t)$. Colors correspond to initial separations $250 \leq r_{0} \leq 1500 \mathrm{~m}$ (see legend in Fig. 3).
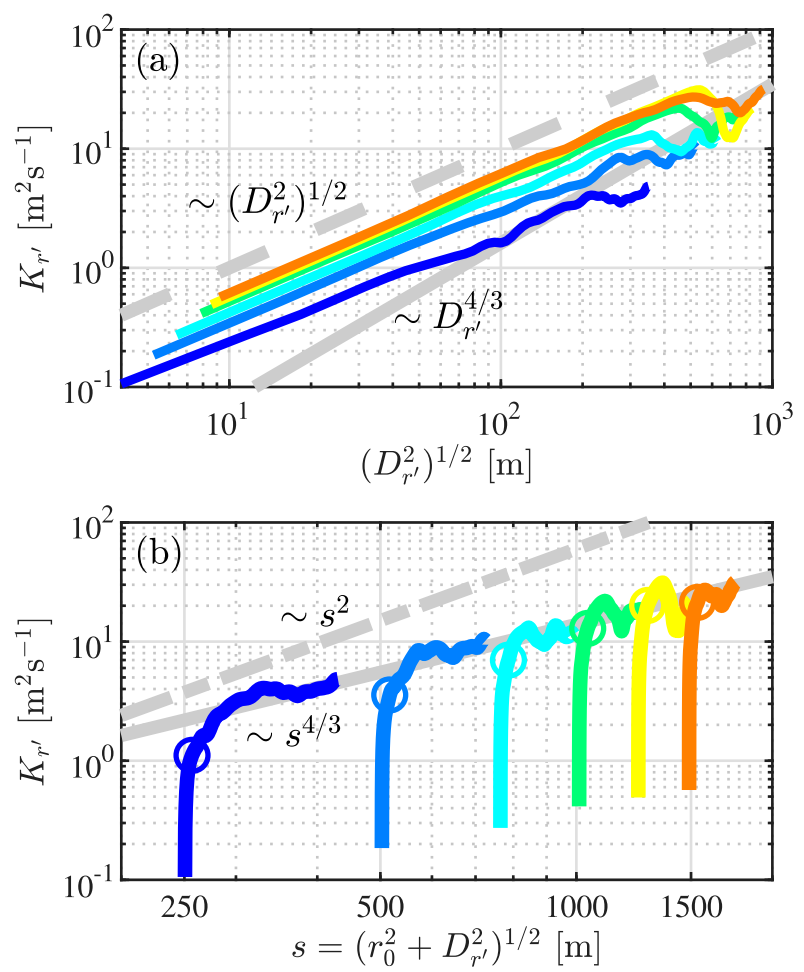

FIG. 6. (a) The EA perturbation diffusivity $K_{r^{\prime}}$ vs (a) EA perturbation dispersion $\left(D_{r^{\prime}}^{2}\right)^{1 / 2}$ and (b) total separation $s=\left(r_{0}^{2}+D_{r^{\prime}}^{2}\right)^{1 / 2}$. Colors correspond to initial separations $250 \leq r_{0} \leq 1500 \mathrm{~m}$ (see legend in Fig. 3). In (a), Batchelor scaling $\sim D_{r^{\prime}}$ (dashed gray) and RO scaling $\sim D_{r^{\prime}}^{4 / 3}$ (gray) are shown. In (b), the RO scaling $\sim s^{4 / 3}$ (thick solid gray) and the enstrophy scaling $\sim s^{2}$ (dash-dotted gray) are shown. Circles in (b) indicate the Batchelor time $t_{B}$.

scaling when the total separation $s=\left(r_{0}^{2}+D_{r^{\prime}}^{2}\right)^{1 / 2}$ is used for the scale (thin gray line in Fig. 6b). The approach to $s^{4 / 3}$ scaling only occurs after the Batchelor time $t_{B}$ for each $r_{0}$ (circles in Fig. $6 \mathrm{~b}$ indicate $t_{B}$ ). In Fig. $6 \mathrm{~b}$, before $t_{B}$ each $r_{0}$ curve has vertical tails due to $s \approx r_{0}$ in the Batchelor regime $\left(D_{r^{\prime}}^{2} \ll r_{0}^{2}\right)$. The diffusivity $K_{r^{\prime}}$ is approaching the same $\sim s^{4 / 3}$ scaling found in previous studies (e.g., Poje et al. 2014). Specifically, the thick gray curve in Fig. $6 \mathrm{~b}$ is very close to the drifter data curve in Fig. 6 of Poje et al. (2014). Using the dispersion $D_{r}$, rather than $s$, results in the same scale dependence because $s^{2} \approx D_{r}^{2}$ (Fig. 4a). Moreover, the dependence of the diffusivity $K_{r}$ on scale $s$ is approximately the same as $K_{r^{\prime}}$ versus $s$ except $K_{r}$ versus $s$ lacks the vertical Batchelor regime tails for each $r_{0}$ as $K_{r}$ lacks an initial Batchelor regime. Although each $r_{0}$ curve appears to approach the same $\mathrm{RO} s^{4 / 3}$ scaling (thin gray line in Fig. 6b), drifter trajectories were too short to definitively observe RO scaling which would have the different $r_{0}$ curves collapse in Fig. 6b. Thus, for this dataset, the scale dependence of the diffusivity shows clear evidence of a Batchelor regime and suggests that the dispersion maybe approaching RO scaling.

As indicated by the circles in Fig. 3c, squared Batchelor velocities $\mathscr{b}^{2}\left(r_{0}\right)$ increase with $r_{0}$ (colored circles in Fig. 7). Overall, there is no consistent $\mathscr{b}^{2}\left(r_{0}\right)$ scaling over the entire 


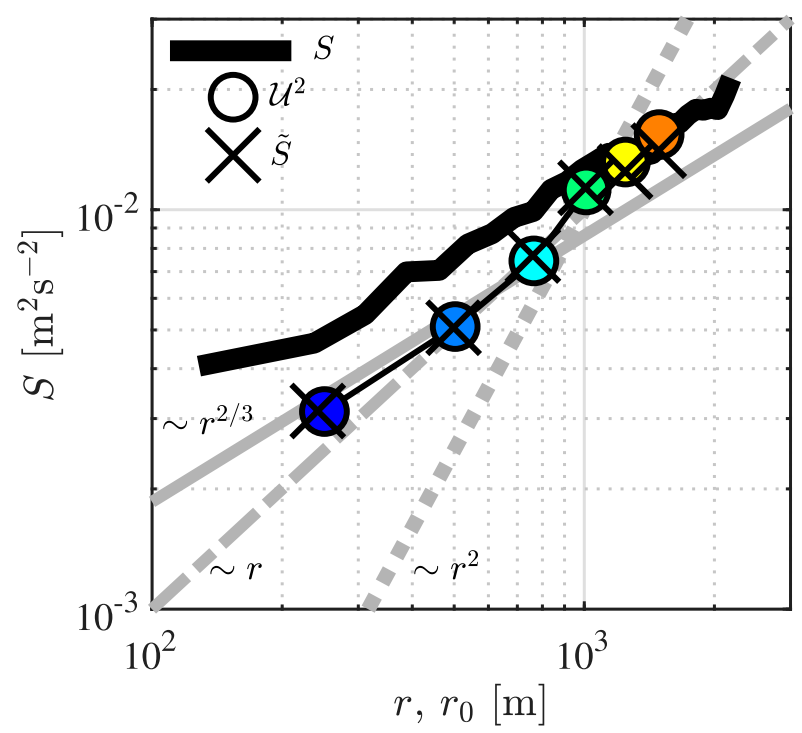

FIG. 7. Drifter pair trajectory derived second-order experiment averaged (EA) structure functions $S$ (thick black) (5) vs $r$. The EA structure function $\tilde{S}$ based on velocity increments from initial drifter pair trajectory times (see text) vs $r$ are shown as " $\mathrm{x}$ " marks. Squared Batchelor velocities $\mathscr{C}^{2}$ (circles) (18) are derived from the Batchelor scaling of $D_{r^{\prime}}^{2}$ and colored according to $r_{0}$ (as in Fig. 3). Energy cascade $\sim r^{2 / 3}$, enstrophy cascade $\sim r^{2}$, and $\sim r$ scalings are shown as solid, dotted gray, dash-dotted lines, respectively.

range of $r_{0}$ and neither enstrophy cascade $\left(\sim r_{0}^{2}\right)$ nor inertial subrange scaling $\left(\sim r_{0}^{2 / 3}\right)$ is indicated (cf. colored dots to solid and dotted gray lines in Fig. 7). The structure function $S(r)$ (black curve in Fig. 7) calculated using (5) for all drifter pair data is $\sim r^{2 / 3}$. For $r \geq 1000 \mathrm{~m}$, the structure function and squared Batchelor velocities are similar, i.e., $S \approx \mathscr{U b}^{2}$. However, for $r \leq 750 \mathrm{~m}, S(r) \neq \mathscr{U}^{2}\left(r_{0}\right)$ despite their theoretical equivalence. Specifically, for $r_{0} \leq 750 \mathrm{~m}, S(r)>\mathscr{C}^{2}\left(r_{0}\right)$ by less than a factor of 2 and the $r_{0}$ scaling for $\mathscr{U}{ }^{2}$ is steeper than the $r$ scaling for $S$. The difference between $\mathscr{C}^{2}$ and $S$ is due to $S$ including all $\left(\approx 10^{4} \mathrm{~s}\right)$ data for each drifter pair trajectory whereas $\mathscr{b ^ { 2 }}$ is based on the only the initial growth which uses the first 5 (600 s) times of each pair trajectory. To verify this, a modified structure function $\tilde{S}$ is calculated using (5) but with only the initial times $(t \leq 600 \mathrm{~s})$ of each drifter pair trajectory. Because $\mathscr{b}^{2}\left(r_{0}\right)$ is found using only the first $600 \mathrm{~s}$ of each pair trajectory, $\mathscr{C}^{2}\left(r_{0}\right)$ should approximately equal the modified structure function $\tilde{S}(r)$ for all $r_{0}$ and $r$. Indeed, the modified structure function $\tilde{S}(r)$, which is essentially Eulerian as the flow has not yet preferentially placed the drifters in convergence zones, is nearly $\mathscr{C}^{2}\left(r_{0}\right)$ (cf. " $\mathrm{x}$ " marks and colored dots in Fig. 7) with largest deviations for $r \geq 1250 \mathrm{~m}$.

Differences between $S(r)$ and $\tilde{S}(r)$ for $r \leq 500 \mathrm{~m}$ are not due to sampling error, rather they are due to drifters preferentially sampling regions of convergence biasing $S(r)$ at small $r$ to larger values relative to the Eulerian estimate (Pearson et al. 2019). The differences are not due to sampling errors as the distributions of $\left\|\delta \mathbf{u}_{m n}\right\|$ used to estimate $S(r)$ and $\tilde{S}(r)$ for $r \leq$ $500 \mathrm{~m}$ are different at the $99 \%$ confidence level according to a Kolmogorov-Smirnov test. Thus, as drifter positions evolve in time, drifters tend to sample regions of surface convergence with larger velocity variance and are thus no longer unbiasedly sampling the flow (as in Pearson et al. 2019).

\section{b. Single release statistics}

We now present the results for drifter statistics averaged over each release (Table 1). Statistics averaged over each release are calculated for the following reasons: 1) to examine the variation in the dispersion between releases, 2) to illustrate the degree to which single release (SR) statistics can differ from EA statistics, and 3) to examine the effect of particular flow events, in particular nonlinear internal waves, on dispersion statistics. In addition to finite sampling effects, the statistics for each release will differ from each other because the flow was not stationary over the 12 releases, i.e., dispersion differences between releases are not just noise but can be due to flow differences. With 12 releases, $12 \mathrm{SR}$ perturbation dispersions $D_{r^{\prime}}^{2}\left(t, r_{0}\right)(9)$ and SR total dispersions $D_{r}^{2}\left(t, r_{0}\right)(8)$ are calculated. Note that some releases do not have drifter pairs in every $r_{0}$ bin. For the SR statistics, $t$ is roughly the time since release (column 2 in Table 1) because drifters for each release were rapidly deployed (within $\approx 35 \mathrm{~min}$ ). Because $\mathrm{SR}$ statistics for each $r_{0}$ are based on fewer drifter pairs $(\sim 20)$ than EA statistics $(\geq 200)$, SR statistics are inherently noisier than EA statistics. However, the number of drifter pairs used in the analysis of SR statistics here is similar to some previously reported oceanographic dispersion statistics (e.g., Ollitrault et al. 2005).

\section{1) THE 13 SEPTEMBER 2017 RELEASE}

To illustrate individual pair (IP) and the single release (SR) dispersion, the dispersion for the 13 September 2017 release (Fig. 1b) is presented. The initial drifter pair number $N_{p}$ for $r_{0}=500 \mathrm{~m}$ is $N_{p}=27$ before dropping dramatically for $t \geq$ $1.5 \times 10^{4} \mathrm{~s}$ (red curve, Fig. 8a). Averaging over each release separately greatly reduces $N_{p}$ for each $r_{0}$ compared to averaging over the entire experiment (i.e., compare this $N_{p}$ to that in Fig. 3a). For this $r_{0}$ with 27 initial pairs, IP perturbation dispersions $r^{\prime 2}$ are variable in time and between drifter pairs (thin blue lines Fig. 8a). For instance, all $r^{\prime 2}$ initially grows in time reaching a local maximum at 5000-8000 s (depending on which $r^{\prime 2}$ ), then dropping for 1000-2000s before increasing again. The variability between $r^{2}$ is considerable with $r^{\prime 2}$ ranging from $\approx 0$ to $5 \times 10^{5} \mathrm{~m}^{2}$ for $t \approx 7000 \mathrm{~s}$.

The time variability for this $r_{0}=500 \mathrm{~m}$ is also evident in the single release (SR) perturbation dispersion $D_{r^{\prime}}^{2}(t)$ (the mean of these $r^{\prime 2}$, thick black curve Fig. 8a) with $D_{r^{\prime}}^{2}$ increasing from 0 at $t=0$ to $0.2 \times 10^{6} \mathrm{~m}^{2}$ at $t=7000 \mathrm{~s}$ then dropping until $t \approx 9000 \mathrm{~s}$ before rising to $\approx 0.4 \times 10^{6} \mathrm{~m}^{2}$ (at $\left.t=1.5 \times 10^{4} \mathrm{~s}\right)$. Overall, for $r_{0}=500 \mathrm{~m}$ the growth of $D_{r^{\prime}}^{2}(t)$ appears to be $t^{2}$ despite the local maximum at $t \approx 7000 \mathrm{~s}$. The variability across individual drifter pairs at time $t$ is quantified by the standard deviation of $\sigma_{D_{J^{\prime}}^{2}}(t)$, e.g., (15) and for this $r_{0}$ scales directly with $D_{r \prime}^{2}(t)$ for $t \leq 1.5 \times$ $10^{4}$ s, i.e., $\sigma_{D_{r^{\prime}}^{2}}(t) \approx D_{r^{\prime}}^{2}(t)$ (e.g., the thin black curve $D_{r^{\prime}}^{2}-\sigma_{D_{r^{\prime}}^{2}}$ in Fig. 8 a is $\stackrel{r^{\prime}}{\approx} 0$ for $t \leq 1.5 \times 10^{4} \mathrm{~s}$ ).

The SR total dispersion $D_{r}^{2}(t)$ for $r_{0}=500 \mathrm{~m}$ shows similar time dependence to the SR perturbation dispersion $D_{r^{\prime}}^{2}(t)$ (cf. thick black curves Figs. $8 \mathrm{a}$ and $8 \mathrm{~b}$ ) but has larger magnitude (note different $y$ scales between Figs. 8a and 8b). Thus, for this 

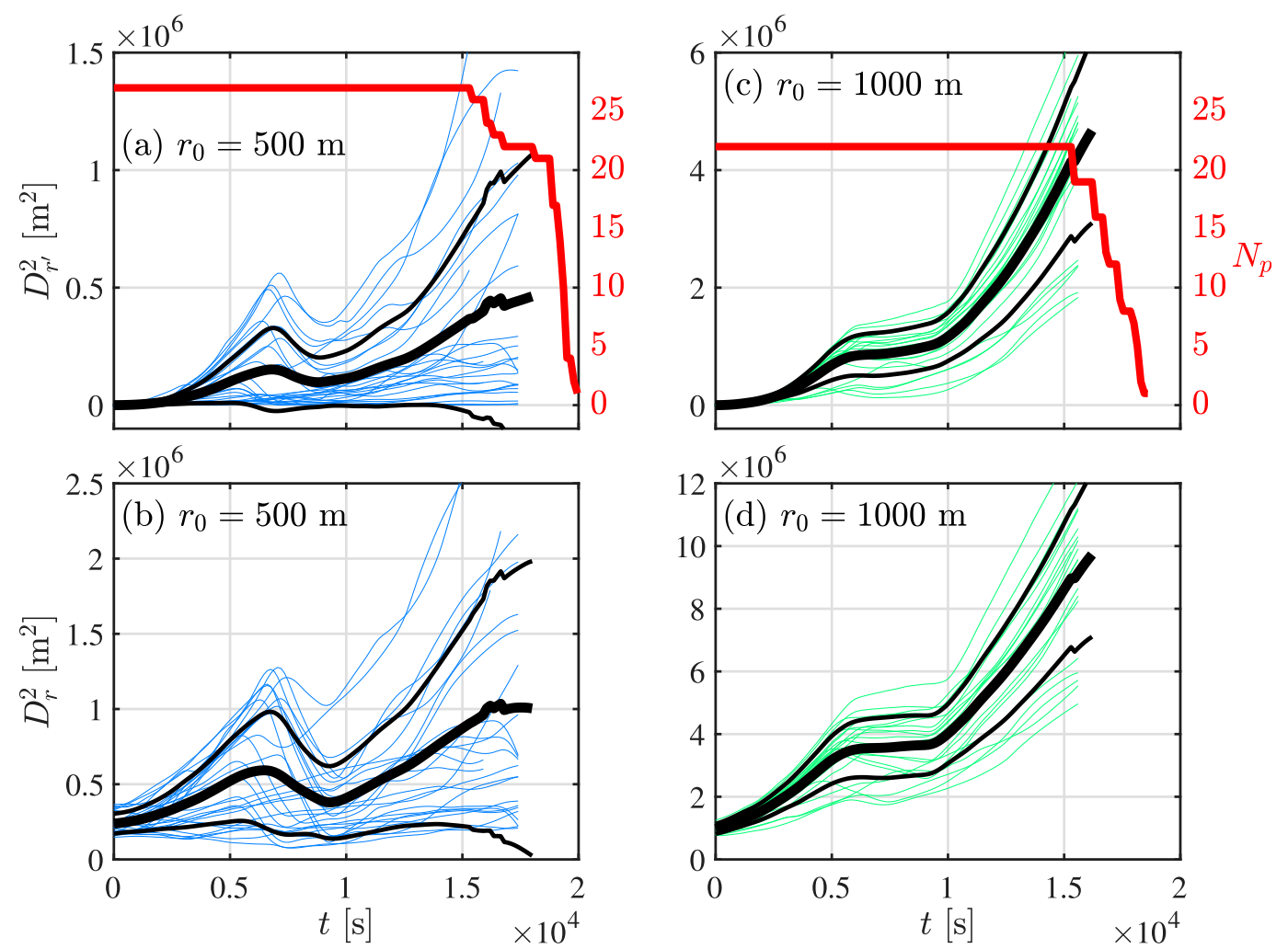

FIG. 8. Dispersion vs time for the 13 Sep 2017 release: (a),(c) perturbation dispersion $D_{r^{\prime}}^{2}(t)$, and (b),(d) total dispersion $D_{r}^{2}(t)$ for (left) $r_{0}=500 \mathrm{~m}$ and (right) $r_{0}=1000 \mathrm{~m}$. Individual pair (IP) dispersion $r^{\prime 2}$ (6) [in (a) and (c)] and IP $r^{2}$ [in (b) and (d)] are colored curves. In each panel, the mean of all pairs (thick black), i.e., the single release statistic, and the mean \pm one standard deviation (thin black) are shown. The number of drifter pairs $N_{p}(t)$ for $r_{0}=$ $500 \mathrm{~m}$ in (a) and $r_{0}=1000 \mathrm{~m}$ in (b) is indicated by the red curve. Means and IP dispersions are shown as long as the number of drifter pairs is within five of the initial number of pairs, i.e., as long as $N_{p}(0)-N_{p}(t) \leq 5(\approx 5 \mathrm{~h}$ for $r_{0}=500 \mathrm{~m}, \approx 4 \mathrm{~h}$ for $r_{0}=1000 \mathrm{~m}$ ). Thus, five IP dispersions are shorter in duration than the mean.

release and $r_{0}, \Phi \neq 0$. For instance, at the local maximum $(t \approx$ $7000 \mathrm{~s}), \Phi \approx 0.2 \times 10^{6} \mathrm{~m}^{2}$, a value greater than $D_{r^{\prime}}^{2}$. Similar to $\sigma_{D^{2}}$, the total dispersion variability across pairs for this $r_{0}=$ $500 \mathrm{~m}$ scales with the total dispersion but offset by the initial total dispersion such that $\sigma_{D_{r}^{2}}(t) \approx D_{r}^{2}(t)-D_{r}^{2}(0)$ (lower thin black curve in Fig. 8b).

For the 13 September 2017 release, the pair number $N_{p}$ for $r_{0}=1000 \mathrm{~m}$ is initially $N_{p}=22$ before dropping when $t \approx$ $1.5 \times 10^{4} \mathrm{~s}$ (red curve Fig. $8 \mathrm{c}$ ). The SR perturbation dispersion $D_{r^{\prime}}^{2}(t)$ for $r_{0}=1000 \mathrm{~m}$ shows similar time dependence to the $r_{0}=500 \mathrm{~m} \mathrm{SR} D_{r^{\prime}}^{2}(t)$. Overall the growth appears quadratic $\left(\sim t^{2}\right)$ but is approximately 8 times larger in magnitude (thick black curves in Figs. $8 \mathrm{a}$ and $8 \mathrm{c}$ ). A point of difference is that $D_{r^{\prime}}^{2}$ for $r_{0}=1000 \mathrm{~m}$ remains constant from 5000 to $10000 \mathrm{~s}$ rather than decreasing like $D_{r^{\prime}}^{2}$ for $r_{0}=500 \mathrm{~m}$. Unlike the variability across pairs for $r_{0}=500 \mathrm{~m}$ in which $\sigma_{D_{t^{\prime}}^{2}} \approx D_{r^{\prime}}^{2}$, for $r_{0}=1000 \mathrm{~m}$ the variability is smaller than the dispersion with $\sigma_{D_{r^{\prime}}^{2}} \approx 0.4 D_{r^{\prime}}^{2}$ (thin black curves Fig. 8c).

The SR total dispersion $D_{r}^{2}(t)$ for $r_{0}=1000 \mathrm{~m}$ shows similar time dependence to the other 13 September 2017 dispersions (thick black curve Fig. 8d). Again, the magnitude of $D_{r}^{2}$ is larger than $D_{r^{\prime}}^{2}$ for $r_{0}=1000 \mathrm{~m}$ (note different $y$ scales between Figs. $8 \mathrm{c}$ and $8 \mathrm{~d}$ ) by a factor of about $2-3$ indicating the $\Phi \neq 0$.
For instance, at $t \approx 7500 \mathrm{~s}$ when $D_{r}^{2}$ and $D_{r^{\prime}}^{2}$ are constant in time, $\Phi \approx 1.6 \times 10^{6} \mathrm{~m}^{2}$ a value more than $2 \times D_{r^{\prime}}^{2}$ at this $t$. Like $D_{r^{\prime}}^{2}$ for this $r_{0}$, the total dispersion variability across pairs $\sigma_{D_{r}^{2}}$ is a fraction of the total dispersion $D_{r}^{2}$. In short, similar to EA dispersion, SR perturbation and total dispersions increase with $r_{0}$ and the total dispersion is larger than the perturbation dispersion for a given $r_{0}$. However, for this release, the SR dispersion is much more variable in time than EA dispersion suggesting that dispersion for single releases may not show a clean scaling law dependence like EA dispersion. Although this is not surprising given that single release statistics are based on fewer drifter pairs, it is illustrative as to how different SR statistics can be from EA statistics.

\section{2) DISPERSION FOR ALL RELEASES}

The perturbation dispersion $D_{r^{\prime}}^{2}$ for all releases is now examined. As such, thick black curves like those in Figs. $8 \mathrm{a}$ and $8 \mathrm{~b}$ are constructed and examined for each release. Similar to the time dependence of $D_{r^{\prime}}^{2}(t)$ for both $r_{0}=500$ and $1000 \mathrm{~m}$ for the 13 September 2017 release (Figs. 8a,b), for all releases and for these $r_{0}$, the initial time dependence of $D_{r^{\prime}}^{2}(t)$ is approximately $\sim t^{2}$ (Figs. 9a,b). Thus, the perturbation dispersion for each release is largely consistent with a Batchelor regime. 


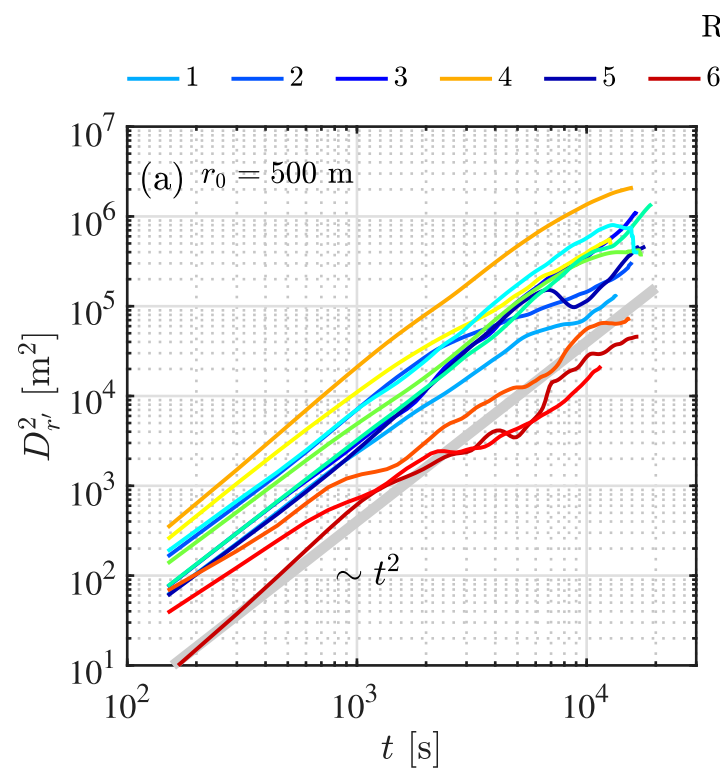

Release

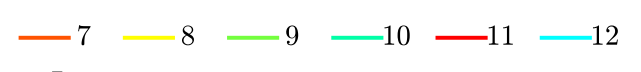

FIG. 9. For each release (colors; consistent with Fig. 1), the single release perturbation dispersion $D_{r^{\prime}}^{2}$ vs time $t$ for (a) $r_{0}=500 \mathrm{~m}$ and (b) $r_{0}=1000 \mathrm{~m}$ initial separations. Red (blue) colors correspond to drifter releases in the northern (southern) portion of the experimental region off of Oceano (Pt. Sal). In (a) and (b), the gray line is $\sim t^{2}$. Statistics are shown only if the number of drifter pairs $N_{p}(t)$ has not dropped by more than 5 from the initial drifter pair number $N_{p}(0)$. Release 4 is not plotted in (b) because this release did not have any drifter pairs with initial separations $r_{0} \geq 1000 \mathrm{~m}$.

The time when $D_{r^{\prime}}^{2}(t)$ departs from Batchelor scaling varies with release. For instance, the two releases (7 and 11) with small initial $D_{r^{\prime}}^{2}$ for $r_{0}=500 \mathrm{~m}$ (red and dark orange curves in Fig. 9a) both depart and grow more slowly than $t^{2}$ at $t \approx$ $1000 \mathrm{~s}$, whereas the departure from $t^{2}$ growth is much later $(t \approx 10000 \mathrm{~s})$ for release 12 (light blue curve in Fig. 9a). Unlike the experiment averaged $D_{r^{\prime}}^{2}(t)$, some releases ( 2 and 5 for $r_{0}=500 \mathrm{~m}$, and 2 for $r_{0}=1000 \mathrm{~m}$ ) transition to faster than $t^{2}$ growth rather than slower. For example, release 2 for $r_{0}=1000 \mathrm{~m}$ is faster than $t^{2}$ at $t=2000 \mathrm{~s}$ before transitioning to slower than $t^{2}$ for $t>4000 \mathrm{~s}$ (blue curve in Fig. 9b). Although the experiment averaged $D_{r^{\prime}}^{2}(t)$ indicates that the Batchelor time $t_{B}$ is associated with departure from $t^{2}$ growth (e.g., Figs. 3c,d), for the individual release $D_{r^{\prime}}^{2}$, a clear pattern of when $D_{r^{\prime}}^{2}$ departs from $t^{2}$ growth is not obvious. For the same $r_{0}$, the Batchelor time $t_{B}=0.25 r_{0} \mathscr{b ^ { - 1 }}$ is inversely proportional to $\mathscr{C}$. Thus, for $r_{0}=500 \mathrm{~m}$, release 4 (orange curve in Fig. 9a), with the largest $2 b^{2}$, ought to depart from $t^{2}$ growth before the other releases and release 6 (dark red curve in Fig. 9a), with the smallest $\mathscr{U}^{2}$, ought to depart from $t^{2}$ growth after the other releases. No $t_{B}$ pattern is apparent likely due to the individual release $D_{r^{\prime}}^{2}$ not representing an ensemble mean and sampling error arising from individual release dispersion based on few drifter pairs.

The SR perturbation dispersion $D_{r^{\prime}}^{2}(t)$ range over the releases is substantial. For instance, initially $(t=150 \mathrm{~s})$ the $r_{0}=$ $500 \mathrm{~m} D_{r^{\prime}}^{2}=12 \mathrm{~m}^{2}$ for release 6 while $D_{r^{\prime}}^{2}=363 \mathrm{~m}^{2}$ for release 4, a factor of 30 difference (Fig. 9a). Thus, squared Batchelor velocities $\mathscr{U}^{2}$ vary by a factor of 30 between releases for $r_{0}=$ $500 \mathrm{~m}$. For $r_{0}=1000 \mathrm{~m}$, the largest initial $D_{r^{\prime}}^{2}\left(688 \mathrm{~m}^{2}\right)$ is
36 times larger than the smallest $D_{r^{\prime}}^{2}\left(19 \mathrm{~m}^{2}\right)$ (Fig. 9b). For both $r_{0}=500$ and $1000 \mathrm{~m}$, the difference between largest and smallest $D_{r^{\prime}}^{2}$ persists for all times with the largest $D_{r^{\prime}}^{2}$ approximately 30 times larger than the smallest $D_{r^{\prime}}^{2}$ for every $t$ (Figs. 9a,b). Although the initial growth of SR dispersion is generally similar to EA dispersion $\left(\sim t^{2}\right)$, the different SR dispersion magnitudes indicate how much tracer dispersion can vary between releases and how much tracer dispersion can differ for a specific release from the ensemble mean. The SR dispersion differences generally correspond to the location of each release, as releases to the north (warm colored curves in Figs. 9a,b) have the smallest $D_{r^{\prime}}^{2}$ and releases to the south (cool colored curves in Figs. 9a,b) generally have the largest $D_{r^{\prime}}^{2}$. For $r_{0}=500 \mathrm{~m}$, release 4 (orange curve with largest $D_{r^{\prime}}^{2}$ ) is an exception as this release was in the northern portion of the domain. However, the initial location of the drifters for this release was in deeper water $(\approx 40 \mathrm{~m})$ than the other releases $(20-30-\mathrm{m}$ depths) (see Fig. 1), potentially affecting the dispersion.

\section{3) SCALING THE DISPERSION WITH $\mathscr{U}^{2}$}

The initial perturbation dispersion for each release generally follows Batchelor scaling $D_{r^{\prime}}^{2}\left(t, r_{0}\right)=\mathscr{C}^{2}\left(r_{0}\right) t^{2}$ (Figs. 9a,b). Here, we examine how the dispersion for each release transitions out of the Batchelor regime and how the dispersion for $t=10^{4}$ s (i.e., $t>t_{B}$ ) depends on $\mathscr{b}^{2}$ and $r_{0}$. Squared Batchelor velocities $\mathscr{U}^{2}\left(r_{0}\right)$ for each release and $r_{0}$ are determined using (18) as the perturbation dispersion for each release is generally consistent with a Batchelor regime. Thus, for $r_{0}=500 \mathrm{~m}, \mathscr{b}^{2}$ is smallest for release 6 and largest for release 4 (initial stacking 
of curves in Fig. 9a). As there are few crossings of the colored curves in Fig. 9a, large $2 b^{2}$ for $r_{0}=500 \mathrm{~m}$ is generally associated with larger later dispersion and small $\mathscr{C}^{2}$ is generally associated with small later dispersion; this is also similar for $r_{0}=1000 \mathrm{~m}$.

Directly comparing the perturbation dispersion $D_{r^{\prime}}^{2}(t)$ at $t=$ $10^{4} \mathrm{~s}$ for each $r_{0}$ and release to the squared Batchelor velocities $\mathscr{C}^{2}$ (Fig. 10) clarifies this relationship. The perturbation dispersion at $10^{4} \mathrm{~s}$ is generally associated with $\mathscr{C b}^{2}$ and the association is largely geographic: northern releases (warm colored dots) have the smallest $\mathscr{C}^{2}$ and $D_{r^{\prime}}^{2}(t)$ at $t=10^{4} \mathrm{~s}$ and southern releases (cool colored dots) have the largest $\mathscr{b ^ { 2 }}$ and $D_{r^{\prime}}^{2}(t)$ at $t=10^{4} \mathrm{~s}$. Although the perturbation dispersion $D_{r^{\prime}}^{2}\left(t=10^{4} \mathrm{~s}\right)$ scales with $\mathscr{b}^{2}$, it is less than what Batchelor dispersion predicts as the colored dots are under dashed black line in Fig. 10 by a factor of approximately $1 / 2$. Thus, as dispersion transitions out of the Batchelor regime, the SR $D_{r^{\prime}}^{2}(t)$ generally slows, relative to continuing as Batchelor, similar to EA dispersion (Fig. 3d).

The initial separation $r_{0}$ affects the later perturbation dispersion. Generally, for a specific release, $D_{r^{\prime}}^{2}\left(t=10^{4} \mathrm{~s}, r_{0}\right)$ increases with $r_{0}$ (larger dots of the same color are above smaller dots in Fig. 10). Thus, the initial separation influences the perturbation dispersion after $10^{4} \mathrm{~s}$ indicating that $r_{0}$-independent RO scaling has not been established. However, the variation in $D_{r^{\prime}}^{2}\left(t=10^{4} \mathrm{~s}, r_{0}\right)$ across $r_{0}$ values for a given release is smaller than differences between releases for the same $r_{0}$, compare the spread of $D_{r^{\prime}}^{2}\left(t=10^{4} \mathrm{~s}\right)$ for the same colored dots to the spread of $D_{r^{\prime}}^{2}\left(t=10^{4} \mathrm{~s}\right)$ for the same sized dots in Fig. 10. Thus, the conditions (day of release) and/or specific release locations (e.g., north or south of Point Sal) can influence the dispersion more than the initial separation such that single release dispersion can vary significantly from the EA dispersion.

\section{Discussion}

\section{a. Influence of internal bores on dispersion}

The experiment averaged, and individual release perturbation dispersion $D_{r^{\prime}}^{2}(t)$, both largely follow Batchelor scaling for $t \lesssim 5000$ s (Figs. 3c and 9). However, some individual releases deviate from Batchelor scaling as seen in Figs. $8 \mathrm{a}$ and $8 \mathrm{c}$ where $D_{r^{\prime}}^{2}(t)$ can stop growing $\left(5000<t<10^{4} \mathrm{~s}\right.$ for $r_{0}=1000 \mathrm{~m}$, Fig. 8c) and even shrink $\left(6000<t<9000 \mathrm{~s}\right.$ for $r_{0}=500 \mathrm{~m}$, Fig. 8a). Via the example of the 13 September 2017 release (Figs. 1b and 8), here, we examine how a particular flow event, namely an onshore propagating nonlinear internal wave (NLIW) affects the dispersion and how NLIWs contribute to deviations from EA Batchelor scaling. NLIWs are known to be significant in the Pt. Sal region (Colosi et al. 2018; McSweeney et al. 2020; Feddersen et al. 2020),

Temperature and velocity are examined from the mooring nearest to the 13 September 2017 drifter release in order to investigate the effect of NLIWs on dispersion. This mooring was located in 30-m water depth offshore of Pt. Sal [magenta asterisk in Figs. $1 \mathrm{a}$ and $1 \mathrm{~b}$ at $(x, y) \approx(-2,0.5) \mathrm{km}]$. As this mooring was inshore of the drifter cluster $\approx 1 \mathrm{~km}$, and bores are known to propagate $\approx 0.25 \mathrm{~m} \mathrm{~s}^{-1}$, we time adjust the mooring $-1 \mathrm{~h}$ to better match the time when the bore passes the mooring and drifter center of mass and therefore to

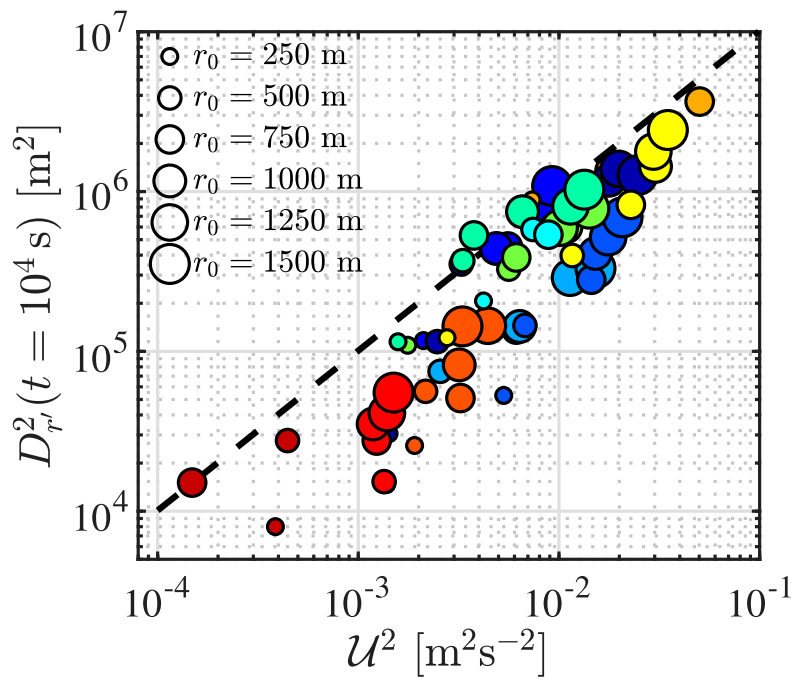

FIG. 10. The single release (SR) perturbation dispersion $D_{r^{\prime}}^{2}(t)$ at time $t=10^{4} \mathrm{~s}$ vs the SR squared Batchelor velocity $\mathscr{\mathscr { b } ^ { 2 }}$. Colors correspond to latitude of the release consistent with Fig. 1. Symbol size corresponds to increasing initial release separation $r_{0}=250$, $500,750, \ldots, 1500 \mathrm{~m}$ (see legend). The dashed black line corresponds to exact Batchelor scaling (3) $D_{r^{\prime}}^{2}(t)=\mathscr{C}^{2} t^{2}$ at $t=10^{4} \mathrm{~s}$.

better highlight the effect of the internal bores on drifter dispersion. During this release, east-west velocities $u$ and temperatures from the 30-m-depth Pt. Sal mooring indicate that a strong NLIW (classified as a warm bore; Colosi et al. 2018) arrived at 1700 UTC (Fig. 11a). Prior to the NLIW arrival ( $<1700$ UTC), surface velocities are offshore $\left(u \approx-0.2 \mathrm{~m} \mathrm{~s}^{-1}\right.$ blues Fig. 11a), deeper $(z<-10 \mathrm{~m})$ velocities are onshore $\left(u \approx 0.1 \mathrm{~m} \mathrm{~s}^{-1}\right)$, and the $16^{\circ} \mathrm{C}$ isotherm (thickest black contour) is near the surface $(z \approx-5 \mathrm{~m})$. After the NLIW arrives, surface temperatures increase by $\approx 1.5^{\circ} \mathrm{C}$ and the $16^{\circ} \mathrm{C}$ isotherm drops to $z \approx-20 \mathrm{~m}$. The NLIW arrival also switches the east-west velocities with surface velocities onshore $\left(u \approx 0.1 \mathrm{~m} \mathrm{~s}^{-1}\right)$ and deeper velocities offshore from 1700 to 1800 UTC. By 1830 UTC, the $16^{\circ} \mathrm{C}$ isotherm has relaxed back to $z \approx-8 \mathrm{~m}$, near its prearrival position, and near surface velocities are again offshore $\left(u \approx-0.05 \mathrm{~m} \mathrm{~s}^{-1}\right)$. Beyond 1830 UTC the influence of the NLIW at the mooring is weak. Examination of the north-south velocities $v$ (not shown) indicates that the NLIW propagates to the east-southeast $\left(18^{\circ}\right.$ from the east).

For the 13 September 2017 release, drifter cross-shore positions $X(t)$ (colored curves, Fig. 11b) indicate that drifters generally move offshore accompanied by cross-shore spreading. The NLIW arrival, found from the maximum cross-shore drifter acceleration (indicated by circles for each drifter in Fig. 11b), interrupts and pauses the cross-shore spreading for $\approx 1 \mathrm{~h}$. From a linear best fit to the timing and cross-shore location of the NLIW arrival, the onshore NLIW propagation speed of this bore is estimated as $0.29 \mathrm{~m} \mathrm{~s}^{-1}$ (slope of circles in Fig. 11b) consistent with regional NLIW phase speeds (Colosi et al. 2018; McSweeney et al. 2020). Thus, the ADCP time offset of $-1 \mathrm{~h}$ used in Fig. 11 effectively places this mooring 

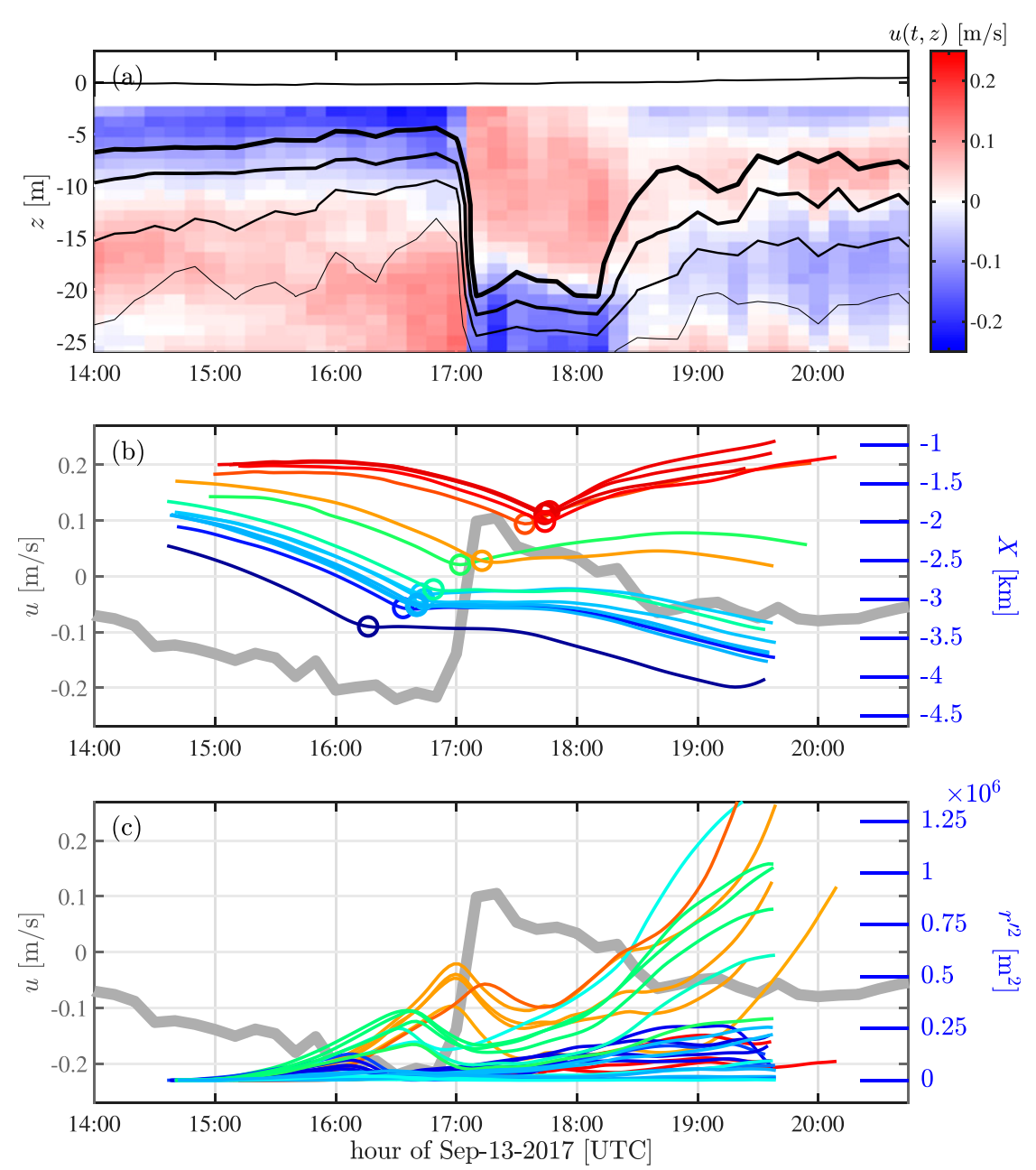

FIG. 11. (a) East-west velocity $u$ (colors) and temperature (black contours at $13^{\circ}, 14^{\circ}, 15^{\circ}$, and $16^{\circ} \mathrm{C}$ ) vs time and depth $z$ at the 30 -m Pt. Sal mooring [magenta asterisk in Figs. 1a and $1 \mathrm{~b}$ at $(x, y) \approx(-2,0.5) \mathrm{km}]$ during the fifth drifter release on 13 Sep 2017. The tide level is indicated by the black curve at $z \approx 0$. (b), (c) Pt. Sal 30-m ADCP near surface ( $z \approx-3 \mathrm{~m}$ ) eastwest velocity $u$ vs time (thick gray curves; left $y$ axis). Colored curves (right $y$ axis) are the 15 individual drifter cross-shore position $X(t)$ in (b) and individual drifter pair perturbation separations $r^{\prime 2}$ for $r_{0}=500 \mathrm{~m}$ vs time in (c) (same as blue curves in Fig. 8a). The trajectories $X(t)$ and individual perturbation dispersions $r^{\prime 2}(t)$ are shown for the first $5 \mathrm{~h}$, the approximate time when the $N_{p}$ for this release and $r_{0}$ has decreased from the initial $N_{p}(0)$ by 6 (see Fig. 8a). Colors correspond to the initial cross-shore drifter position in (b) and the initial drifter pair cross-shore midpoint in (c). Reds denote positions closer to shore, and blues denote farther offshore positions. Mooring times are offset by $-1 \mathrm{~h}$ to better align mooring and drifter times as this mooring was onshore of the drifters.

$\approx 1000 \mathrm{~m}$ farther offshore (at $x \approx-3 \mathrm{~km}$ ) near the center of drifter cross-shore center of mass.

Prior to NLIW arrival, drifter velocities are consistent with cross-shore surface mooring velocities (gray curve Figs. 11b,c) with the most offshore drifters (blue curves, Fig. 11b) having the largest offshore velocities (most negatively sloped curves) and the most onshore drifters have the weakest $\left(\approx 0 \mathrm{~m} \mathrm{~s}^{-1}\right)$ offshore velocities (red curves, Fig. 11b). This is reflected in the surface mooring velocity (gray curve, Fig. 11b) which becomes more negative as the bore approaches the mooring. Thus, before bore arrival at the most offshore drifter $(\approx 1615$ UTC), cross-shore drifter spreading is associated with diverging $(d u / d x>0)$ cross-shore velocities and results in quickly growing $r_{0}=500 \mathrm{~m}$ individual separations $r^{\prime 2}(t)$ (blue curves in Fig. 8a reproduced as colored curves versus UTC time in Fig. 11c). The faster than linear initial growth for all $r^{\prime 2}(t)$ before 1615 UTC appears Batchelor-like, i.e., $\sim t^{2}$. Although individual perturbation separations $r^{\prime 2}$ are due to both cross- and alongshore position differences (6), for this release, examining the cross-shore mooring velocities $u$ and cross-shore drifter 
positions $X(t)$ explains the general features of $r^{2}$ as the bore is propagating nearly due east.

For drifters separated in the cross-shore, the effect of the NLIW is sequential with the NLIW arrival halting and reversing the growth of individual perturbation dispersions $r^{2}$ first for the most offshore pairs (blue curves in Fig. 11c) and later for more onshore pairs (orange curves). When averaged this effect results in the local maxima of $D_{r^{\prime}}^{2}(t)$ at $t \approx 7000 \mathrm{~s}$ (thick black curve Fig. 8a). The details of the process begin with the NLIW passing the most offshore drifter at $\approx 1600$ UTC which results in the drifter $X(t)$ accelerating onshore (indicated by a circle in darkest blue curve in Fig. 11b) halting offshore movement. After the NLIW passes the most offshore drifter but before reaching the other drifters, the cross-shore separation between the most offshore and the other drifters decreases because the most offshore drifter is stationary in the crossshore and the others are moving offshore toward it. Thus, for drifter pairs containing the most offshore drifter, perturbation separations $r^{\prime 2}(t)$ increase and decrease before and after, respectively, NLIW arrival (blue curves Fig. 11c) indicated by local maxima in $r^{\prime 2}(t)$ a little after 1600 UTC. The NLIW passes the most onshore drifters at $\approx 1745$ UTC (red circles in Fig. 11b), $1.5 \mathrm{~h}$ after passing the most offshore drifter. Thus, the most onshore drifter pairs have the most time for $r^{\prime 2}(t)$ to grow before the bore arrives. This results in the largest $r^{\prime 2}$ maximum at $\approx 1700$ UTC for pairs with cross-shore initial separations (orange curves in Fig. 11c). For onshore pairs with alongshore initial separations, $r^{\prime 2}$ is much smaller (red curves in Fig. 11c).

After this NLIW passes all the drifters ( $>1800$ UTC), the cross-shore positions $X(t)$ begin spreading (Fig. 11b) similarly to before NLIW arrival. The most offshore drifters (blue curves) move offshore (consistent with mooring velocities for 1830-2100 UTC) whereas the most onshore drifters move shoreward. Thus, the spreading is again due to $d u / d x(>0)$ and results in quickly growing $r^{\prime 2}(t)$ similar to, and an approximate continuation of, the initial growth (Fig. 11c). This is reflected in Fig. 10 where the perturbation dispersion $D_{r^{\prime}}^{2}$ at $t=10^{4} \mathrm{~s}$ for all $r_{0}$ for this release (darkest blue dots with $\mathscr{b}^{2} \approx 0.02 \mathrm{~m}^{2} \mathrm{~s}^{-2}$ ) is similar to but smaller (by approximately $50 \%$ ) than the initial squared Batchelor velocity prediction. To summarize, NLIWs act to pulse the perturbation dispersion $D_{r^{\prime}}^{2}$ by quickly growing, then stopping and reversing individual separations after which $D_{r^{\prime}}^{2}$ grows similarly to its initial growth. Although the effect is large in the short term, overall for times longer than a few hours the effect is fleeting as the dispersion generally returns to the initial Batchelor growth. Thus, this NLIW does not appear to be large contributors to the dispersion on time scales longer than a few hours.

\section{b. Comparison to previous work}

For the release-averaged and most individual releases, the perturbation dispersion is consistent with Batchelor scaling $D_{r^{\prime}}^{2}=\mathscr{C}^{2}\left(r_{0}\right) t^{2}$ for $t<t_{B}$ with the Batchelor time $t_{B}$ (16) increasing with initial drifter separation $r_{0}$ in accordance with theory and previous studies (e.g., Ouellette et al. 2006). Batchelor scaling is well established for various nonoceanographic laboratory and numerical investigations of inertial subrange turbulent dispersion (e.g., Salazar and Collins 2009) as well as proposed for dispersion within an atmospheric simulation (Haszpra et al. 2012). For oceanographic dispersion, Batchelor scaling has not been examined, however, other dispersive scalings have been identified and examined. Because the dispersive scaling is linked to the background turbulence wavenumber spectra [(17); e.g., Foussard et al. 2017], identifying the correct dispersive scaling is critical to properly inferring the turbulence responsible for the dispersion. Batchelor scaling may not have been identified or examined in previous oceanographic studies due to limited data, examining incompatible time scales, and investigating a dispersion statistic $\left(D_{r}^{2}\right)$ for which Batchelor scaling is less clear.

Some oceanographic observations and modeling studies are suggestive of Batchelor scaling. For a few drifters repeatedly released in the Santa Barbara Channel, resulting in less than 75 total pairs, Ohlmann et al. (2012) found $D_{r}^{2} \sim t^{2}$ for $5<t<84 \mathrm{~h}$. In this study, a thorough examination of Batchelor scaling was not possible as only one small $r_{0} \approx 7.5 \mathrm{~m}$ was considered. In modeling studies of coastal Southern California, for a single $r_{0} \approx 500 \mathrm{~m}$ and $t \leq 1$ day, the dispersion $D_{r}^{2}$ is similar to $t^{2}$ in a 40-m resolution model (Dauhajre et al. 2019) and $D_{r}^{2} \sim t^{2}$ for drifters released within $2 \mathrm{~km}$ of the shoreline in a $250-\mathrm{m}$ resolution model (Romero et al. 2013). In a model of the Adriatic Sea, the dispersion is approximately $D_{r}^{2}(t) \sim t^{2}$ for $r_{0} \approx 1$ and $5 \mathrm{~km}$ and $t>10$ days (Haza et al. 2008). In this study, time or space smoothing affects the results. On the inner shelf of the Gulf of Mexico, a modified perturbation dispersion $\left\langle r^{2}\right\rangle-\langle r\rangle^{2}$ shows $\sim t^{2}$ growth for $t \lesssim 3 \times 10^{4}$ s (Roth et al. 2017). In these previous studies, Batchelor scaling was not examined. Some previous studies that have found ballistic $D_{r}^{2} \sim t^{2}$ dispersion (LaCasce and Ohlmann 2003; Roth et al. 2017) suggested that it might be due to horizontal shear dispersion. However, the $t^{2}$ growth rate from this mechanism requires pure uniform horizontal shear (LaCasce 2008) which the drifter trajectories here do not exhibit (Figs. 1a,b). Moreover, the structure function in a uniform shear scales as $S(r) \sim r^{2}$ for drifters released similarly to the drifters here. The requirement of pure shear is strong as any small-scale turbulence, in addition to the uniform shear, results in $D_{r}^{2} \sim t^{3}$ growth (LaCasce 2008).

A Batchelor regime may not have been identified in previous oceanographic observations because the drifter sampling rates were too slow or the time scales analyzed were too long to properly resolve a Batchelor regime. For example, in some studies (Ollitrault et al. 2005; Koszalka et al. 2009), the drifter sampling resolution (daily) was much greater than, the Batchelor time found here $\left(t_{B} \approx 1 \mathrm{~h}\right.$, green + in Fig. $\left.3 \mathrm{c}\right)$ for the $r_{0} \approx 1 \mathrm{~km}$ initial separation considered. In Lumpkin and Elipot (2010), where $r_{0} \approx 1 \mathrm{~km}$, the sampling rate was faster (1-2h) but still insufficient to resolve $t \lesssim 1 \mathrm{~h}$ and hence a Batchelor regime for this $r_{0}$. In Beron-Vera and LaCasce (2016), where $r_{0} \approx 1 \mathrm{~km}$, the drifter sampling rate (15 min) was fast enough to resolve a Batchelor regime, however, only times $(\geq 2.4 \mathrm{~h}$ ) beyond the Batchelor time were analyzed. In contrast to these studies, both the temporal resolution (5 min) and time scales ( $\leqslant$ hours) considered here were sufficient to resolve a Batchelor regime.

More importantly, even with sampling rates fast enough to resolve a Batchelor regime (e.g., Ohlmann et al. 2012; 
Beron-Vera and LaCasce 2016), in most studies (e.g., Lumpkin and Elipot 2010; Ohlmann et al. 2012; Romero et al. 2013; Beron-Vera and LaCasce 2016, etc.) identifying a Batchelor regime may have been hindered because the total dispersion $D_{r}^{2}(t)$ was analyzed rather than the perturbation dispersion $D_{r^{\prime}}^{2}(t)$. Ouellette et al. (2006) discusses how $D_{r}^{2}(t)$ may hinder the correct dispersive scalings due to including initial separation effects, here quantified as $\Phi$. How $D_{r}^{2}(t)$ or $D_{r^{\prime}}^{2}(t)$ can lead to different conclusions is examined by considering the experiment averaged $D_{r}^{2}$ and $D_{r^{\prime}}^{2}$ for $r_{0}=1 \mathrm{~km}$ (green curve in Fig. $3 \mathrm{~b}$ and Fig. 4a). For $t \leq 10^{4} \mathrm{~s}, D_{r}^{2}(t)$ is well fit by the exponential $r_{0}^{2} \exp [t /(5 \mathrm{~h})]$ (green and dash-dotted curves, respectively, in Fig. 12). Previous oceanographic studies (e.g., Ollitrault et al. 2005; Koszalka et al. 2009; Ohlmann et al. 2012) have also found initial $D_{r}^{2} \sim \exp (t / \tau)$ scaling with various $e$-folding times $\tau$. For instance, in the Santa Barbara Channel for $r_{0}=7.5 \mathrm{~m}, D_{r}^{2} \sim \exp (t)$ for $t<5 \mathrm{~h}$ with an $e$-folding time of $\tau=0.9 \mathrm{~h}$ (Ohlmann et al. 2012) as well as for $r_{0}=1 \mathrm{~km}$ in the Nordic Sea for $t \leq 2$ days with a much larger $e$-folding time $\tau \approx$ $12 \mathrm{~h}$ (Koszalka et al. 2009). Because $D_{r}^{2} \sim \exp (t)$ is associated with a $k^{-3}$ wavenumber spectra (Lin 1972), $D_{r}^{2} \sim \exp (t)$ suggests that the dispersion may be due to a $2 \mathrm{D}$ turbulence enstrophy cascade. According to the theory, $e$-folding times are independent of $r_{0}$ in contrast to exponential fits to observed $D_{r}^{2}\left(t, r_{0}\right)$ (colored curves in Fig. 4a) for which $\tau$ increases with $r_{0}$. Rather than exponential growth, for $r_{0}=1 \mathrm{~km}$ the offset perturbation dispersion $r_{0}^{2}+D_{r^{\prime}}^{2}(t)$ is well fit by offset Batchelor scaling $r_{0}^{2}+\mathscr{b}^{2} t^{2}$ for $t \leq 5000 \mathrm{~s}$ (red and black curves in Fig. 12) before transitioning to RO scaling for $t>6000 \mathrm{~s}$ (red and dashed black curves in Fig. 12). Thus, for $t>6000 \mathrm{~s}, r_{0}^{2}+D_{r^{\prime}}^{2}(t)$ suggests that the dispersion is due to $k^{-5 / 3}$ rather than $k^{-3}$ turbulence as incorrectly suggested by the $D_{r}^{2}(t)=r_{0}^{2} \exp (t / \tau)$ fit valid up to $t \approx 12000 \mathrm{~s}$. Here, $D_{r}^{2}(t)$ is well approximated by an exponential $r_{0}^{2} \exp (t / \tau)$ because initially $r_{0}^{2} \exp (t / \tau) \approx r_{0}^{2}(1+t / \tau)$ and $D_{r}^{2}(t)=r_{0}^{2}+D_{r^{\prime}}^{2}(t)+\Phi(t)$, see $(10)$ and (11), with $\Phi \sim t$ and $\Phi>D_{r^{\prime}}^{2}$ (green curve in Fig. 4b) for $t \lesssim 3000 \mathrm{~s}$. To properly estimate the dispersive scaling(s), and therefore correctly infer the background turbulent wavenumber spectra, the perturbation dispersion $D_{r^{\prime}}^{2}(t)$ must be examined (e.g., Ouellette et al. 2006) as correct dispersive scalings are more easily identified in $D_{r^{\prime}}^{2}(t)$ rather than $D_{r}^{2}(t)$ which can have $\Phi \neq 0$ effects.

Note that rather than transitioning directly from Batchelor to RO scaling, the offset perturbation dispersion (red curve Fig. 12) slows after Batchelor but before RO scaling similar to laboratory dispersion (Ouellette et al. 2006). Here, this slowing is due to the RO fit including an $r_{0}$ offset since the fit for the perturbation dispersion is $D_{r^{\prime}}^{2}(t) \approx C_{1} r_{0}^{2}+C_{2} t^{3}$ with $C_{1} \neq 0$ whereas for theoretical RO scaling $D_{r^{\prime}}^{2}$ would not have the $r_{0}$ term and $C_{1}=0$. However, in accordance with inertial subrange theory (Batchelor 1950), for large enough $t$, the $t^{3}$ term is eventually much larger than $r_{0}^{2}$ term.

\section{Summary}

GPS-equipped surface drifters were repeatedly deployed on the Inner Shelf off of Pt. Sal, CA, in water depths $\leq 40 \mathrm{~m}$. Relative dispersion statistics were calculated from 1998 drifter

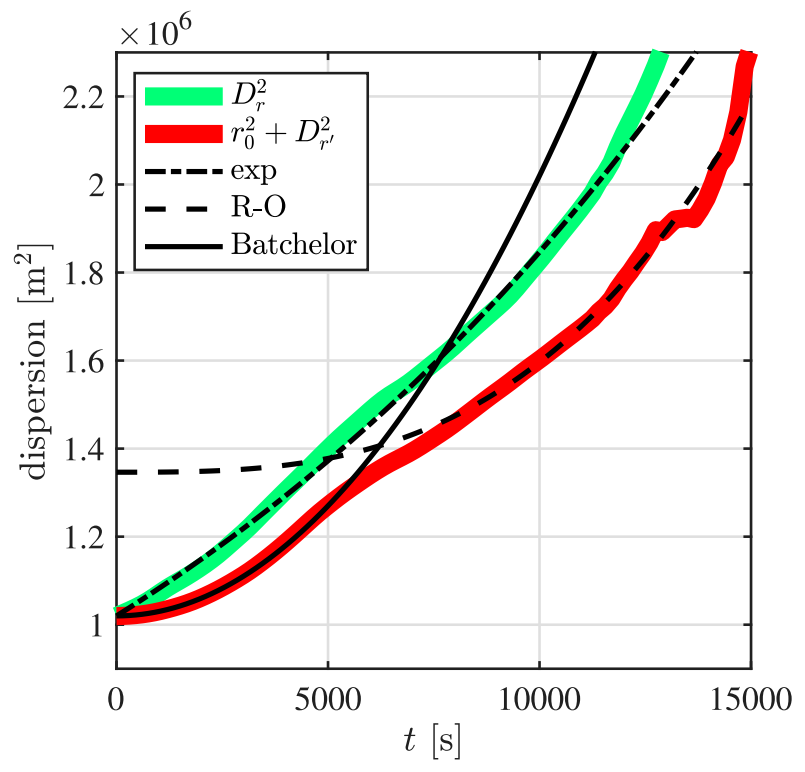

FIG. 12. The experiment averaged (EA) total dispersion $D_{r}^{2}(t)$ (green) and offset EA perturbation dispersion $r_{0}^{2}+D_{r^{\prime}}^{2}(t)$ (red) vs time $t$ for the 13 Sep 2017 release and $r_{0}=1000 \mathrm{~m}$. Solid and dashed black curves are fits to the offset perturbation dispersion: (solid) Batchelor $r_{0}^{2}+\mathscr{Q} b^{2}\left(r_{0}\right) t^{2}$ for $t<5000 \mathrm{~s}$, and (dashed) RO $\left(1.3 r_{0}\right)^{2}+$ $\left(2.5 \times 10^{-7} \mathrm{~m}^{2} \mathrm{~s}^{-3}\right) t^{3}$ for $6000 \leq t \leq 15000 \mathrm{~s}$. Dash-dotted curve is the exponential fit $r_{0}^{2} \exp [t /(4.7 \mathrm{~h})]$ for $t<10^{4} \mathrm{~s}$ to $D_{r}^{2}(t)$.

pairs from 12 releases of $\approx 18$ drifters per release. Unlike most previous studies that focus on the dispersion $D_{r}^{2}(8)$, here, the perturbation dispersion $D_{r \prime}^{2}(9)$ was also analyzed. Diffusivities $K_{r}(12)$ and perturbation diffusivities $K_{r^{\prime}}(13)$ were additionally calculated and analyzed. Statistics were presented for the entire experiment (EA statistics) and each single release (SR statistics) for $t \lesssim 4 \mathrm{~h}$ and for initial drifter separations $250 \leq r_{0} \leq 1500 \mathrm{~m}$.

The EA perturbation dispersion follows Batchelor scaling $D_{r^{\prime}}^{2}\left(t, r_{0}\right)=\mathscr{U}^{2}\left(r_{0}\right) t^{2}$ (3) for 1000-3000 s for $r_{0} \geq 750 \mathrm{~m}$. Consistent with theory, both the duration of Batchelor scaling $t_{B}\left(r_{0}\right)$ and squared Batchelor velocities $\mathscr{C b}^{2}\left(r_{0}\right)$ increase with initial separation $r_{0}$. EA squared Batchelor velocities $\mathscr{C b}^{2}\left(r_{0}\right)$ for $r_{0}=1500 \mathrm{~m}$ are 5 times greater than $\mathscr{Q b}^{2}$ for $r_{0}=$ $250 \mathrm{~m}$. For $r_{0} \leq 1000 \mathrm{~m}, \mathrm{EA} \mathscr{C}^{2}\left(r_{0}\right)$ are nearly equivalent to the unbiased velocity structure function $\tilde{S}$ calculated from initial drifter pair trajectories. In contrast to inertial subrange scaling where $\mathscr{U b}^{2} \sim r_{0}^{2 / 3}$, and enstrophy cascade scaling where $\mathscr{b}^{2} \sim r_{0}^{2}$, here $\mathscr{C b}^{2}\left(r_{0}\right)$ does not have a single power-law dependence for all $r_{0}$. After Batchelor scaling, i.e., $t>t_{B}$, scale $s=\left(r_{0}^{2}+D_{r^{\prime}}^{2}\right)^{1 / 2}$ dependent EA perturbation diffusivities suggest approach to the classic Richardson 4/3-law $K_{r^{\prime}} \sim s^{4 / 3}$ consistent with RO dispersion (1). For the $r_{0}$ values considered here, the EA $D_{r^{\prime}}^{2}(t)$ at times larger than $t_{B}$ is smaller than but correlated with the initial Batchelor scaling. Specifically, the dispersion at $t=10^{4} \mathrm{~s}$ is approximately $50 \%$ of that predicted by Batchelor scaling. This indicates that the dispersion does not transfer directly from Batchelor to RO scaling but rather slows when transitioning out of the Batchelor regime. For each release and all 
$r_{0}$, the SR $D_{r^{\prime}}^{2}$ generally follows Batchelor scaling. For a given $r_{0}$ and time $t$, the SR $D_{r^{\prime}}^{2}\left(t, r_{0}\right)$ variation between releases is significant-varying by almost a factor of 100 . This variation is attributed to squared Batchelor velocities $\mathscr{C}^{2}$ that are much larger ( $>10$ times) in the southern region of the domain (off Pt. Sal) compared to the northern region (off Oceano). Each release also slows when transitioning out of the Batchelor regime as the SR perturbation dispersion at $t=10^{4} \mathrm{~s}$ is correlated with but less than the Batchelor scaling prediction.

For an individual release, a NLIW modulated (enhancing and then reducing) the dispersion. Potential reasons why previous studies did not investigate a Batchelor regime include the following. 1) The focus was on time scales that were too long. 2) Only one initial separation $r_{0}$ was considered. 3) Most importantly, investigating only the dispersion $D_{r}^{2}(t)$ that can scale differently than the perturbation dispersion $D_{r^{\prime}}^{2}(t)$ for which Batchelor scaling is clear. Here, Batchelor scaling is not evident in $D_{r}^{2}(t)$ as the correlations between initial and later separations $\Phi(t)$ scale as $\sim t$ for short times. Thus, previous studies investigating $D_{r}^{2}(t)$ are potentially aliased by initial separation effects that are not present in $D_{r^{\prime}}^{2}(t)$. Thus, analysis of both $D_{r}^{2}$ and $D_{r}^{\prime 2}$ is critical in order to accurately determine the dispersion power law time dependence and therefore the underlying turbulent velocity wavenumber spectra.

Acknowledgments. The Office of Naval Research supported this research through Grants N00014-5-1-2631 (SIO) and N0001418WX00229 (NPS). This work was also supported by the National Science Foundation (OCE-1459389). For the Point Sal fieldwork, Bill Boyd, Greg Boyd, Tucker Freismuth, Casey Gon, Matt Gough, Rob Grenzeback, Derek Grimes, Ami Hansen, Paul Jessen, Michael Kovatch, Paul Lenz, Aaron Morrone, Andy O'Neill, Lucian Parry, Brett Pickering, Greg Sinnett, Kent Smith, Marla Stone, Ata Suanda, Jim Thomson, Brian Woodward, and Keith Wyckoff are acknowledged for their help in deployment and recovery. We thank John Colosi for generously providing mooring and ADCP data. The authors thank Derek Grimes, Thomas Zdyrski, and Xiaodong $\mathrm{Wu}$ for providing useful feedback. The authors thank three anonymous reviewers for numerous constructive comments that improved the manuscript.

Data availability statement. Inner-shelf dynamics experiment drifter and mooring data are archived at UC San Diego Library Digital Collections (https://doi.org/10.6075/J0WD3Z3Q).

\section{REFERENCES}

Babiano, A., C. Basdevant, and R. Sadourny, 1985: Structure functions and dispersion laws in two-dimensional turbulence. J. Atmos. Sci., 42, 941-949, https://doi.org/10.1175/1520-0469(1985)042<0941: SFADLI $>2.0 . \mathrm{CO} ; 2$.

Batchelor, G. K., 1950: The application of the similarity theory of turbulence to atmospheric diffusion. Quart. J. Roy. Meteor. Soc., 76, 133-146, https://doi.org/10.1002/qj.49707632804.

Beron-Vera, F. J., and J. H. LaCasce, 2016: Statistics of simulated and observed pair separations in the Gulf of Mexico. J. Phys. Oceanogr., 46, 2183-2199, https://doi.org/10.1175/JPO-D-150127.1.
Bitane, R., H. Homann, and J. Bec, 2012: Time scales of turbulent relative dispersion. Phys. Rev., 86E, 045302, https://doi.org/ 10.1103/PhysRevE.86.045302.

Brown, J., J. MacMahan, A. Reniers, and E. Thornton, 2009: Surf zone diffusivity on a rip-channeled beach. J. Geophys. Res., 114, C11015, https://doi.org/10.1029/2008JC005158.

Colosi, J. A., N. Kumar, S. H. Suanda, T. M. Freismuth, and J. H. MacMahan, 2018: Statistics of internal tide bores and internal solitary waves observed on the inner continental shelf off Point Sal, California. J. Phys. Oceanogr., 48, 123-143, https:// doi.org/10.1175/JPO-D-17-0045.1.

Cressman, J. R., J. Davoudi, W. I. Goldburg, and J. Schumacher, 2004: Eulerian and Lagrangian studies in surface flow turbulence. New J. Phys., 6, 53, https://doi.org/10.1088/1367-2630/6/1/053.

D'Asaro, E. A., and Coauthors, 2018: Ocean convergence and the dispersion of flotsam. Proc. Natl. Acad. Sci. USA, 115, 11621167, https://doi.org/10.1073/pnas.1718453115.

Dauhajre, D. P., J. C. McWilliams, and L. Renault, 2019: Nearshore Lagrangian connectivity: Submesoscale influence and resolution sensitivity. J. Geophys. Res. Oceans, 124, 5180-5204, https://doi.org/10.1029/2019JC014943.

Davis, R. E., 1985: Drifter observations of coastal surface currents during CODE: The method and descriptive view. J. Geophys. Res., 90, 4741-4755, https://doi.org/10.1029/JC090iC03p04741.

Dräger-Dietel, J., K. Jochumsen, A. Griesel, and G. Badin, 2018: Relative dispersion of surface drifters in the Benguela upwelling region. J. Phys. Oceanogr., 48, 2325-2341, https:// doi.org/10.1175/JPO-D-18-0027.1.

Feddersen, F., J. H. MacMahan, T. M. Freismuth, M. K. Gough, and M. Kovatch, 2020: Inner-shelf vertical and alongshore temperature variability in the subtidal, diurnal, and semidiurnal bands along the central California coastline with headlands. J. Geophys. Res. Oceans, 125, e2019JC015347, https:// doi.org/10.1029/2019JC015347.

Foussard, A., S. Berti, X. Perrot, and G. Lapeyre, 2017: Relative dispersion in generalized two-dimensional turbulence. J. Fluid Mech., 821, 358-383, https://doi.org/10.1017/jfm.2017.253.

Gutiérrez, P., and S. Aumaître, 2016: Clustering of floaters on the free surface of a turbulent flow: An experimental study. Eur. J. Mech., 60, 24-32, https://doi.org/10.1016/ j.euromechflu.2016.06.009.

Haszpra, T., P. Kiss, T. Tél, and I. Jánosi, 2012: Advection of passive tracers in the atmosphere: Batchelor scaling. Int. J. Bifurcation Chaos, 22, 1250241, https://doi.org/10.1142/ S0218127412502410.

Haza, A. C., A. C. Poje, T. M. Özgökmen, and P. Martin, 2008: Relative dispersion from a high-resolution coastal model of the Adriatic Sea. Ocean Modell., 22, 48-65, https://doi.org/ 10.1016/j.ocemod.2008.01.006.

Huntley, H. S., B. L. Lipphardt Jr., G. Jacobs, and A. D. Kirwan Jr., 2015: Clusters, deformation, and dilation: Diagnostics for material accumulation regions. J. Geophys. Res. Oceans, 120, 6622-6636, https://doi.org/10.1002/2015JC011036.

Kolmogorov, A. N., 1941: The local structure of turbulence in an incompressible viscous fluid for very large Reynolds numbers. Dokl. Akad. Nauk SSSR, 30, 299-303.

Koshel, K. V., D. V. Stepanov, E. A. Ryzhov, P. Berloff, and V. I. Klyatskin, 2019: Clustering of floating tracers in weakly divergent velocity fields. Phys. Rev., 100E, 063108, https:// doi.org/10.1103/PhysRevE.100.063108.

Koszalka, I., J. H. LaCasce, and K. A. Orvik, 2009: Relative dispersion in the Nordic Seas. J. Mar. Res., 67, 411-433, https:// doi.org/10.1357/002224009790741102. 
Kumar, N., and Coauthors, 2021: The Inner Shelf Dynamics Experiment. Bull. Amer. Meteor. Soc., https://doi.org/10.1175/ BAMS-D-19-0281.1, in press.

LaCasce, J. H., 2008: Statistics from Lagrangian observations. Prog. Oceanogr., 77, 1-29, https://doi.org/10.1016/j.pocean.2008.02.002.

_ , 2016: Estimating Eulerian energy spectra from drifters. Fluids, 1, 33, https://doi.org/10.3390/fluids1040033.

, and A. Bower, 2000: Relative dispersion in the subsurface North Atlantic. J. Mar. Res., 58, 863-894, https://doi.org/ 10.1357/002224000763485737.

— , and C. Ohlmann, 2003: Relative dispersion at the surface of the Gulf of Mexico. J. Mar. Res., 61, 285-312, https://doi.org/ 10.1357/002224003322201205.

Lin, J.-T., 1972: Relative dispersion in the enstrophy-cascading inertial range of homogeneous two-dimensional turbulence. J. Atmos. Sci., 29, 394-396, https://doi.org/10.1175/15200469(1972)029<0394:RDITEC > 2.0.CO;2.

Lumpkin, R., and S. Elipot, 2010: Surface drifter pair spreading in the North Atlantic. J. Geophys. Res., 115, C12017, https:// doi.org/10.1029/2010JC006338.

Maximenko, N., J. Hafner, and P. Niiler, 2012: Pathways of marine debris derived from trajectories of Lagrangian drifters. Mar. Pollut. Bull., 65, 51-62, https://doi.org/10.1016/j.marpolbul.2011.04.016.

McSweeney, J. M., and Coauthors, 2020: Observations of shoaling nonlinear internal bores across the central California inner shelf. J. Phys. Oceanogr., 50, 111-132, https://doi.org/10.1175/ JPO-D-19-0125.1.

Novelli, G., C. M. Guigand, C. Cousin, E. H. Ryan, N. J. M. Laxague, H. Dai, B. K. Haus, and T. M. Özgökmen, 2017: A biodegradable surface drifter for ocean sampling on a massive scale. J. Atmos. Oceanic Technol., 34, 2509-2532, https:// doi.org/10.1175/JTECH-D-17-0055.1.

Obukhov, A. M., 1941: On the distribution of energy in the spectrum of turbulent flow. Izv. Akad. Nauk SSSR. Ser. Geogr. Geofiz., 5, 453-466.

Ohlmann, J. C., J. H. LaCasce, L. Washburn, A. J. Mariano, and B. Emery, 2012: Relative dispersion observations and trajectory modeling in the Santa Barbara Channel. J. Geophys. Res., 117, C05040, https://doi.org/10.1029/2011JC007810.

Okubo, A., 1971: Oceanic diffusion diagrams. Deep-Sea Res., 18, 789-802, https://doi.org/10.1016/0011-7471(71)90046-5.

- 1980: Diffusion and Ecological Problems: Mathematical Models. Biomathematics Series, Vol. 10, Springer-Verlag, $254 \mathrm{pp}$.

Ollitrault, M., C. Gabillet, and A. C. de Verdière, 2005: Open ocean regimes of relative dispersion. J. Fluid Mech., 533, 381407, https://doi.org/10.1017/S0022112005004556.

Ouellette, N. T., H. Xu, M. Bourgoin, and E. Bodenschatz, 2006: An experimental study of turbulent relative dispersion models. New J. Phys., 8, 109, https://doi.org/10.1088/1367-2630/8/6/109.

Pearson, J., B. Fox-Kemper, R. Barkan, J. Choi, A. Bracco, and J. C. McWilliams, 2019: Impacts of convergence on structure functions from surface drifters in the Gulf of Mexico. J. Phys. Oceanogr., 49, 675-690, https://doi.org/10.1175/JPO-D-180029.1.

Poje, A. C., and Coauthors, 2014: Submesoscale dispersion in the vicinity of the Deepwater Horizon spill. Proc. Natl. Acad. Sci. USA, 111, 12 693-12 698, https://doi.org/10.1073/pnas.1402452111.

, T. M. Özgökmen, D. J. Bogucki, and A. D. Kirwan, 2017: Evidence of a forward energy cascade and Kolmogorov selfsimilarity in submesoscale ocean surface drifter observations. Phys. Fluids, 29, 020701, https://doi.org/10.1063/1.4974331.
Poulain, P.-M., 1999: Drifter observations of surface circulation in the Adriatic Sea between December 1994 and March 1996. J. Mar. Syst., 20, 231-253, https://doi.org/10.1016/ S0924-7963(98)00084-0.

_ _ , and R. Gerin, 2019: Assessment of the water-following capabilities of CODE drifters based on direct relative flow measurements. J. Atmos. Oceanic Technol., 36, 621-633, https://doi.org/10.1175/JTECH-D-18-0097.1.

Richardson, L. F., 1926: Atmospheric diffusion shown on a distance-neighbour graph. Proc. Roy. Soc. London, 110A, 709-737, https://doi.org/10.1098/rspa.1926.0043.

Romero, L., Y. Uchiyama, J. C. Ohlmann, J. C. McWilliams, and D. A. Siegel, 2013: Simulations of nearshore particle-pair dispersion in Southern California. J. Phys. Oceanogr., 43, 1862-1879, https://doi.org/10.1175/JPO-D-13-011.1.

Roth, M. K., J. MacMahan, A. Reniers, T. M. Özgökmen, K. Woodall, and B. Haus, 2017: Observations of inner shelf cross-shore surface material transport adjacent to a coastal inlet in the northern Gulf of Mexico. Cont. Shelf Res., 137, 142-153, https://doi.org/10.1016/j.csr.2016.12.017.

Rupolo, V., 2007: A Lagrangian-based approach for determining trajectories taxonomy and turbulence regimes. J. Phys. Oceanogr., 37, 1584-1609, https://doi.org/10.1175/JPO3038.1.

Rypina, I. I., I. Kamenkovich, P. Berloff, and L. J. Pratt, 2012: Eddy-induced particle dispersion in the near-surface North Atlantic. J. Phys. Oceanogr., 42, 2206-2228, https://doi.org/ 10.1175/JPO-D-11-0191.1.

Salazar, J. P., and L. R. Collins, 2009: Two-particle dispersion in isotropic turbulent flows. Annu. Rev. Fluid Mech., 41, 405-432, https://doi.org/10.1146/annurev.fluid.40.111406.102224.

Sawford, B. L., P. K. Yeung, and J. F. Hackl, 2008: Reynolds number dependence of relative dispersion statistics in isotropic turbulence. Phys. Fluids, 20, 065111, https://doi.org/ 10.1063/1.2946442.

Spydell, M. S., F. Feddersen, R. T. Guza, and W. E. Schmidt, 2007: Observing surf-zone dispersion with drifters. J. Phys. Oceanogr., 37, 2920-2939, https://doi.org/10.1175/2007JPO3580.1.

_ _ _ a and J. Macmahan, 2019: The effect of drifter GPS errors on estimates of submesoscale vorticity. J. Atmos. Oceanic Technol., 36, 2101-2119, https://doi.org/10.1175/JTECH-D19-0108.1.

Suanda, S. H., F. Feddersen, M. S. Spydell, and N. Kumar, 2018: The effect of barotropic and baroclinic tides on threedimensional coastal dispersion. Geophys. Res. Lett., 45, 11 235-11 246, https://doi.org/10.1029/2018GL079884.

Subbaraya, S., A. Breitenmoser, A. Molchanov, J. Muller, C. Oberg, D. A. Caron, and G. S. Sukhatme, 2016: Circling the seas: Design of Lagrangian drifters for ocean monitoring. IEEE Rob. Autom. Mag., 23, 42-53, https://doi.org/10.1109/MRA.2016.2535154.

Taylor, G. I., 1922: Diffusion by continuous movements. Proc. London Math. Soc., S2-20, 196-212, https://doi.org/10.1112/ plms/s2-20.1.196.

Yaremchuk, M., and E. F. Coelho, 2015: Filtering drifter trajectories sampled at submesoscale resolution. IEEE J. Oceanic Eng., 40, 497-505, https://doi.org/10.1109/JOE.2014.2353472.

Young, W. R., P. B. Rhines, and C. J. R. Garrett, 1982: Shear-flow dispersion, internal waves and horizontal mixing in the ocean. J. Phys. Oceanogr., 12, 515-527, https://doi.org/10.1175/15200485(1982)012<0515:SFDIWA $>2.0 . \mathrm{CO} ; 2$.

Zhurbas, V., and I. S. Oh, 2004: Drifter-derived maps of lateral diffusivity in the Pacific and Atlantic Oceans in relation to surface circulation patterns. J. Geophys. Res., 109, C05015, https://doi.org/10.1029/2003JC002241. 